\title{
Wavelet construction of Generalized Multifractional processes
}

\author{
Antoine Ayache, Stéphane Jaffard and Murad S. Taqqu
}

\begin{abstract}
We construct Generalized Multifractional Processes with Random Exponent (GMPREs). These processes, defined through a wavelet representation, are obtained by replacing the Hurst parameter of Fractional Brownian Motion by a sequence of continuous random processes. We show that these GMPREs can have the most general pointwise Hölder exponent function possible, namely, a random Hölder exponent which is a function of time and which can be expressed in the strong sense (almost surely for all $t$ ), as a liminf of an arbitrary sequence of continuous processes with values in $[0,1]$.
\end{abstract}

\section{Introduction and main results}

Fractional Brownian Motion (FBM) on $\mathbb{R}^{d}$ with Hurst parameter $H \in(0,1)$ will be denoted $\left\{B_{H}(t)\right\}_{t \in \mathbb{R}^{d}}$. This continuous and nowhere differentiable process can be represented for every $t \in \mathbb{R}^{d}$, as the Wiener integral

$$
B_{H}(t)=\int_{\mathbb{R}^{d}} \frac{e^{i t \cdot \xi}-1}{|\xi|^{H+d / 2}} d W(\xi)
$$

where $t \cdot \xi$ denotes the usual scalar product and $|\cdot|$ the usual Euclidian norm. Throughout this article, the complex-valued Brownian measure $d W$ is chosen in a way that all the processes and the fields we consider are real-valued (see for example [6] or [8]). FBM was introduced in 1940 by Kolmogorov as a way to generate Gaussian "spirals" in Hilbert space [28] but its systematic study started with the famous 1968 paper of Mandelbrot and Van Ness [31]. It has been studied since then by many authors, such as Adler, Falconer, Kahane,

2000 Mathematics Subject Classification: Primary: 60G18, 60G17. Secondary: 65T16. Keywords: Fractional brownian motion, generalized multifractional brownian motion, Hölder regularity. 
Samorodnitsky, Talagrand and Taqqu [2, 17, 27, 36, 37]. FBM shares many nice properties with the Wiener process but also has more flexibility since it includes it. This is why FBM has now become a standard model: it is used in many areas such as hydrology, economics, finance, physics and telecommunications $[1,17,26,36]$. The monograph of Doukhan, Oppenheim and Taqqu [16] offers a systematic treatment of FBM, as well as an overview of different areas of applications.

The process $\left\{B_{H}(t)\right\}_{t \in \mathbb{R}^{d}}$ is $H$-self-similar and with stationary increments [36]. $H$-self-similarity means that for all constants $a>0$, the processes $\left\{B_{H}(a t)\right\}_{t \in \mathbb{R}^{d}}$ and $\left\{a^{H} B_{H}(t)\right\}_{t \in \mathbb{R}^{d}}$ have the same finite-dimensional distributions. Because of its self-similarity, FBM has been frequently used for the modelling of fractal signals. However, in many situations, such a modelling is not realistic because, the pointwise Hölder exponent of a fractal signal evolves in time, while that of FBM remains constant (see for instance $[5,9,10,20,26,30,34,35])$. The pointwise Hölder exponent of a stochastic process $\{X(t)\}_{t \in \mathbb{R}^{d}}$ will be denoted $\left\{\alpha_{X}(t)\right\}_{t \in \mathbb{R}^{d}}$. It measures the local variation of $\{X(t)\}_{t \in \mathbb{R}^{d}}$ : the larger it is the more regular is $\{X(t)\}_{t \in \mathbb{R}^{d}}$. Since we focus here on the pointwise Hölder exponents of continuous and nowhere differentiable stochastic processes, $\left\{\alpha_{X}(t)\right\}_{t \in \mathbb{R}^{d}}$ has values in $[0,1]$ and can be defined for every $t \in \mathbb{R}^{d}$, as

$$
\alpha_{X}(t, \omega)=\sup \left\{\alpha, \limsup _{h \longrightarrow 0} \frac{|X(t+h, \omega)-X(t, \omega)|}{|h|^{\alpha}}=0\right\} .
$$

Multifractional processes were introduced in order to overcome the limitations resulting from the constancy of the pointwise Hölder exponent of FBM. Their main features are the following: a) they extend FBM, b) almost all their trajectories are continuous, c) their pointwise Hölder exponents can be prescribed and are allowed to evolve in time.

The paradigmatic example of a Multifractional process is Multifractional Brownian Motion (MBM). It was introduced in [13, 34] and can be obtained by replacing the Hurst parameter in (1.1) by a continuous function $t \mapsto H(t)$ with values in $(0,1)$. The MBM $\left\{B_{H(t)}(t)\right\}_{t \in \mathbb{R}^{d}}$ will be denoted $\{P(t)\}_{t \in \mathbb{R}^{d}}$. The assumption on the continuity of $H(\cdot)$ is needed to insure the continuity of $\{P(t)\}_{t \in \mathbb{R}^{d}}[6]$. Moreover, when $H(\cdot)$ is a $\beta$-Hölder function (i.e for all $t_{1}$ and $t_{2}$, one has $\left|H\left(t_{1}\right)-H\left(t_{2}\right)\right| \leq c\left|t_{1}-t_{2}\right|^{\beta}$ ) satisfying the technical assumption

$$
\sup _{t} H(t)<\beta
$$

then MBM satisfies the following two important properties: 
- At any point $t, \mathrm{MBM}$ is Locally Asymptotically Self-Similar with index $H(t)$ [13]. This means that

(1.4) $\lim _{r \rightarrow 0^{+}} \operatorname{law}\left\{\frac{P(t+r u)-P(t)}{r^{H(t)}}\right\}_{u \in \mathbb{R}^{d}}=\operatorname{law}\left\{B_{H(t)}(u)\right\}_{u \in \mathbb{R}^{d}}$,

where $\left\{B_{H(t)}(u)\right\}_{u \in \mathbb{R}^{d}}$ is an FBM with Hurst parameter $H(t)$.

- The pointwise Hölder exponent of MBM can be prescribed via the function $H(\cdot)$. More precisely, for all $t$, one has almost surely,

$$
\alpha_{P}(t, \omega)=H(t)
$$

MBM can be viewed as an extension of FBM for at least the following two reasons:

- MBM reduces to an FBM when the function $H(\cdot)$ is constant.

- Relation (1.4) means that at each point $t$, there is an FBM with parameter $H(t)$ tangent to the MBM. Thus MBM looks locally like an FBM whose Hurst parameter changes with the location. We refer to the recent works of Falconer $[18,19]$ for an extensive study of the notion of tangent process.

Relation (1.5) has been established in the weak sense: for all $t$ almost surely in $[13,34]$. By "in the weak sense" we mean that it holds on a set of probability 1 which may depend on $t$. We will prove here that Relation (1.5) remains true in the strong sense, almost surely for all $t$, that is it holds on a set of probability 1 which does not depend on $t$. This together with the continuity of the function $H(\cdot)$ imply that the pointwise Hölder exponent of MBM, namely, the function $t \mapsto \alpha_{P}(t, \omega)$, cannot be discontinuous. This is a strong limitation, both from an applied and theoretical perspective. Indeed, on one hand, the pointwise Hölder exponent of a real-life signal appears often erratic and on the other hand, Proposition 1.1 below implies that the class of pointwise Hölder exponents of continuous and nowhere differentiable functions is much larger than that of continuous functions.

Proposition $1.1([3, \mathbf{1 5}, \mathbf{2 3}])$ A function $H(\cdot)$ with values in $[0,1]$ is the pointwise Hölder exponent of a continuous and nowhere differentiable complex-valued function $f(\cdot)$ defined over $\mathbb{R}^{d}$, if and only if there is a sequence of continuous functions $\left(H_{n}(\cdot)\right)_{n \in \mathbb{N}}$ such that for every $t$,

$$
H(t)=\liminf _{n \rightarrow \infty} H_{n}(t) .
$$


In view of this result, a function which is the liminf of a sequence of continuous functions with values in $[0,1]$ will be called a pointwise Hölder exponent of the most general form. This is a slight abuse of language since we consider only pointwise Hölder exponents of continuous functions $f(\cdot)$. If we drop the assumption of continuity for $f(\cdot)$, then we could consider bounded functions $f(\cdot)$, but the most general form of pointwise Hölder exponents of functions that are merely bounded, is not known.

Observe that while the functions $H_{n}(\cdot), n \in \mathbb{N}$, in Proposition 1.1, are continuous, their liminf, namely $H(\cdot)$ may be discontinuous, in fact, quite irregular, as for instance, the indicator function of any open set of $\mathbb{R}^{d}[7]$ or the indicator function of the irrationals [23].

Continuous functions $f(\cdot)$ with a most general pointwise Hölder exponent $H(\cdot)$ have been obtained in a deterministic context in $[23,15]$. They are very pecular and therefore cannot be used in any realistic simulation. This is why the problem of finding a natural probabilistic construction for such functions has been raised in [24]. A "natural probabilistic construction" means a stochastic process extending a standard model such as Fractional Brownian Motion, that is, a Multifractional process.

Note that it is not useful to force discontinuities in the pointwise Hölder exponent of an MBM by simply taking a discontinuous $H(\cdot)$ defined as $H(t, \omega)=\sum_{k=1}^{n} g_{k}(t) \chi_{A_{k}}(\omega)$, where one of the $g_{k}$ is discontinuous at some point $t_{0}$. Indeed, while $H(\cdot, \omega)$ is discontinuous, the trajectories of the $\operatorname{MBM}\left\{B_{H(t, \omega)}(t)\right\}$ will also be discontinuous with probability 1 ([6, Proposition 3.1]). A more refined approach is needed to construct a continuous process with a most general pointwise Hölder exponent.

The problem mentioned above has motivated the introduction of the Generalized Multifractional Brownian Motion (GMBM) $\{X(t)\}_{t \in \mathbb{R}^{d}}[5,7,8]$. This continuous Gaussian process is obtained, roughly speaking, by replacing the Hurst parameter $H$ in the harmonizable representation of FBM (1.1) by an admissible sequence $\left(H_{n}(\cdot)\right)_{n \in \mathbb{N}}$ of Lipschitz functions in the sense of Definition 2.1 below. For every $t \in \mathbb{R}^{d}$, the GMBM $X(t)$ is defined, as the Wiener integral

$$
X(t)=\int_{\mathbb{R}^{d}} K\left(t,\left\{H_{n}(t)\right\}, \xi\right) d W(\xi),
$$

with

$$
K\left(t,\left\{H_{n}(t)\right\}, \xi\right)=\sum_{n=0}^{\infty} \frac{\left(e^{i t \cdot \xi}-1\right)}{|\xi|^{H_{n}(t)+d / 2}} \widehat{f}_{n-1}(\xi),
$$

where $\left\{\widehat{f}_{n-1}(\cdot)\right\}_{n \in \mathbb{N}}$ is a sequence of smoothing functions specified in Definition 2.2 . 
To study the GMBM, Ayache [5] also introduced the Generalized Multifractional Field

$$
\begin{aligned}
Y(x, y) & =\int_{\mathbb{R}^{d}} K\left(x,\left\{H_{n}(y)\right\}, \xi\right) d W(\xi) \\
& =\int_{\mathbb{R}^{d}}\left[\sum_{n=0}^{\infty} \frac{\left(e^{i x \cdot \xi}-1\right)}{|\xi|^{H_{n}(y)+d / 2}} \widehat{f}_{n-1}(\xi)\right] d W(\xi), \quad x, y \in \mathbb{R}^{d},
\end{aligned}
$$

and then set

$$
X(t)=Y(t, t), \quad t \in \mathbb{R}^{d}
$$

The GMBM can be viewed as an extension of FBM and MBM at least for the following two reasons:

- GMBM reduces to an MBM with parameter $H(\cdot)$ (respectively an FBM with parameter $H$ ) when all the functions $H_{n}(\cdot)$ are equal to a same function $H(\cdot)$ (respectively to a same real $H$ ).

- Under some technical conditions on $\left(H_{n}(\cdot)\right)_{n \in \mathbb{N}}$, GMBM is Locally Asymptotically Self-Similar with index

$$
H(t)=\liminf _{n \rightarrow \infty} H_{n}(t)
$$

at each point $t$ (see [7, Proposition 3]).

Ayache [5] studied the pointwise Hölder regularity of the GMBM in dimension $d=1$ and for (non-random) functions $H_{n}(\cdot)$ taking values in a compact interval in $(0,1)$. Extending results of [7] and [8], Ayache showed that the pointwise Hölder exponent of the GMBM satisfies in the weak sense, for every $t \in \mathbb{R}^{1}$, almost surely,

$$
\alpha_{X}(t, \omega)=\liminf _{n \rightarrow \infty} H_{n}(t) .
$$

By using different techniques, we will prove here a much stronger result, namely:

(a) Relation (1.10) holds in the strong sense and when $t \in \mathbb{R}^{d}$.

(b) This happens also when the deterministic functions $H_{n}(t)$ are replaced by random functions $S_{n}(t, \omega)$ which need not to be independent of the white noise $d W$. 
The process obtained by replacing the non-random functions $H_{n}(t)$ by stochastic processes $S_{n}(t, \omega)$ will be denoted $\{Z(t)\}_{t \in \mathbb{R}^{d}}$. It will be called the Generalized Multifractional Process with Random Exponent (GMPRE) since it extends not only GMBM but also the process introduced in [6]. Observe that the GMPRE uses an anticipative representation since the random kernel $\left\{K\left(t,\left\{S_{n}(t, \omega)\right\}, \xi\right)\right\}_{\xi \in \mathbb{R}^{d}}$ is not adapted to the natural filtration of $\{W(\xi)\}_{\xi \in \mathbb{R}^{d}}$. It is an open problem to determine whether there exists an non-anticipative representation formulated as an Itô integral.

Using Proposition 6.1 which is a stochastic version of Proposition 1.1, we will prove that the GMPRE can have a pointwise Hölder exponent of the most general form:

Theorem 1.1 Let $\left(\left\{S_{n}(t)\right\}_{t \in \mathbb{R}^{d}}\right)_{n \in \mathbb{N}}$ be a sequence of stochastic processes whose trajectories are, with probability 1 , continuous functions with values in $[0,1]$. Then one can construct an almost sure continuous process $\{Z(t)\}_{t \in \mathbb{R}^{d}}$, called a GMPRE, which satisfies almost surely for all $t_{0} \in \mathbb{R}^{d}$,

$$
\limsup _{t \rightarrow t_{0}} \frac{\left|Z(t)-Z\left(t_{0}\right)\right|}{\left|t-t_{0}\right|}=\infty
$$

and whose pointwise Hölder exponent $\left\{\alpha_{Z}(t)\right\}_{t \in \mathbb{R}^{d}}$ satisfies, in the strong sense, (almost surely for every $t$ )

$$
\alpha_{Z}(t, \omega)=\liminf _{n \rightarrow \infty} S_{n}(t, \omega) .
$$

Our goal is to prove Theorem 1.1. Here are some consequences of this theorem.

Corollary $\mathbf{1 . 1}$ (a) Almost all the trajectories of the process $\{Z(t)\}_{t \in \mathbb{R}^{d}}$ are nowhere differentiable functions.

(b) Relations (1.5) and (1.10) hold in the strong sense almost surely for all $t$.

(c) The class of pointwise Hölder exponents of GMPREs coincides with the class of pointwise Hölder exponents of continuous and nowhere differentiable processes.

Part (a) follows from (1.11), Part (b) from (1.12) and Part (c) from Theorem 1.1 and Proposition 6.1 below.

In view of Part (c) one can restrict oneself to GMPREs if one is interested in a continuous and nowhere differentiable process with the most general pointwise Hölder exponent possible. Recall that while the processes $\left\{S_{n}(t)\right\}_{t \in \mathbb{R}^{d}}, n \in \mathbb{N}$, in Theorem 1.1 are continuous, the resulting pointwise Hölder exponent $\left\{\alpha_{Z}(t)\right\}_{t \in \mathbb{R}^{d}}$ can be discontinuous and rather erratic. 
As indicated above, we have restricted our study to processes with continuous trajectories because the class of pointwise Hölder exponents of bounded functions has not been identified even in the deterministic case, and therefore, it is premature to construct bounded processes with a most general exponent.

To study the GMPRE, we will introduce the corresponding field called the Generalized Multifractional Field with Random Exponent (GMFRE). The GMFRE is to the GMPRE as $Y$ is to $X$ in (1.8) and (1.9). The main ingredient of the proof of Theorem 1.1 will be the following theorem.

Theorem 1.2 Let $\{V(x, y)\}_{(x, y) \in \mathbb{R}^{d} \times \mathbb{R}^{d}}$ be a GMFRE. Then almost surely, for all $t_{0} \in \mathbb{R}^{d}$,

$$
\limsup _{t \rightarrow t_{0}} \frac{\left|V\left(t, t_{0}\right)-V\left(t_{0}, t_{0}\right)\right|}{\left|t-t_{0}\right|}=\infty
$$

and the pointwise Hölder exponent at $t_{0}$ of the process $\left\{V\left(t, t_{0}\right)\right\}_{t \in \mathbb{R}^{d}}$ equals $\liminf _{n \rightarrow \infty} S_{n}\left(t_{0}\right)$.

The paper is structured as follows. We present the construction of the GMPRE and GMFRE in Section 2. Theorem 1.2 is proved in Sections 3 and 4, Theorem 1.1 in Section 5 and auxiliary results are proved in Section 6 . Section 7 contains some final remarks.

\section{Construction of the GMFRE and the GMPRE}

Here are the basic ideas underlying the construction of the Generalized Multifractional Field with Random Exponent (GMFRE) and the Generalized Multifractional Process with Random Exponent (GMPRE):

- Start with Fractional Brownian Motion (1.1).

- Replace the single $H$ by a sequence $\left\{H_{n}\right\}$ as in (1.6).

- Allow the sequence $\left\{H_{n}\right\}$ to be random (call it $\left\{S_{n}\right\}$ ) and not necessarily independent of the measure $W$.

- Use a wavelet representation instead of the representation (1.6).

- Focus on the GMFRE $\{V(x, y)\}_{(x, y) \in \mathbb{R}^{d} \times \mathbb{R}^{d}}$ and define the GMPRE to be the diagonal process $Z(t)=V(t, t), t \in \mathbb{R}^{d}$.

We first introduce the wavelet representation. Throughout this article

$$
\left\{\psi_{l, j, k}(x) ; 1 \leq l \leq 2^{d}-1, j \in \mathbb{Z}, k \in \mathbb{Z}^{d}\right\}
$$

will denote a Lemarié-Meyer wavelet basis of the Hilbert space $L^{2}\left(\mathbb{R}^{d}\right)$ (see for example [32]). This basis has the following properties: 
(a) The functions $\psi_{l, j, k}$ are real valued and belong to the Schwartz class $S\left(\mathbb{R}^{d}\right)$. Recall that $S\left(\mathbb{R}^{d}\right)$ is the space of infinitely differentiable functions $f$ whose partial derivatives of any order decrease at infinity, faster than any polynomial, i.e for any multi-index $\alpha=\left(\alpha_{1}, \ldots, \alpha_{d}\right) \in \mathbb{N}^{d}$ and for every integer $m \in \mathbb{N}$, there exist $c>0$ (a constant that generally depends on $\alpha$ and $m$ ), such that for any $\xi \in \mathbb{R}^{d}$,

$$
\left|\partial^{\alpha} f(\xi)\right|=\left|\frac{\partial^{\alpha_{1}+\ldots+\alpha_{d}} f}{\left(\partial \xi_{1}\right)^{\alpha_{1}} \ldots\left(\partial \xi_{d}\right)^{\alpha_{d}}}(\xi)\right| \leq c(1+|\xi|)^{-m} .
$$

(b) The functions $\psi_{l, j, k}$ are generated by dilations and translations of a finite number of functions. Namely, one has

$$
\psi_{l, j, k}(x)=2^{j d / 2} \psi_{l}\left(2^{j} x-k\right)
$$

Thus the Fourier transforms of the $\psi_{l, j, k}$ also belong to the Schwartz class and satisfy

$$
\widehat{\psi}_{l, j, k}(\xi)=2^{-j d / 2} e^{-i k \cdot 2^{-j} \xi} \widehat{\psi}_{l}\left(2^{-j} \xi\right) .
$$

(c) For all $l, j, k$ one has

$$
\operatorname{supp} \widehat{\psi}_{l, j, k} \subset D_{j+2} \backslash D_{j}
$$

where

$$
D_{j}=\left[-\frac{2^{j+1} \pi}{3}, \frac{2^{j+1} \pi}{3}\right]^{d}
$$

and $D_{j+2} \backslash D_{j}=\left\{\xi \in D_{j+2} ; \xi \notin D_{j}\right\}$.

In view of (2.3) and (2.4), high values of $j$ correspond to high "frequencies" $\left|\xi_{i}\right|, i=1, \ldots, d$. Since the Fourier transform is an isometry of $L^{2}\left(\mathbb{R}^{d}\right)$, the system

$$
\left\{\widehat{\psi}_{l, j, k}(-\xi) ; 1 \leq l \leq 2^{d}-1, j \in \mathbb{Z}, k \in \mathbb{Z}^{d}\right\}
$$

is also an orthonormal bases for $L^{2}\left(\mathbb{R}^{d}\right.$ ) (up to the multiplicative factor $(2 \pi)^{-d / 2}$ that we will neglect). We use the basis

$$
\left\{\widehat{\psi}_{l, j, k}(-\xi)\right\}_{l, j, k}=\left\{{\widehat{\hat{\psi}_{l, j, k}(\xi)}}_{l, j, k}\right.
$$

instead of $\left\{\widehat{\psi}_{l, j, k}(\xi)\right\}_{l, j, k}$ for convenience, in order to avoid having to add constantly the complex conjugate sign. 
To motivate the wavelet representation, we will start with the random field $\{Y(x, y)\}_{(x, y) \in \mathbb{R}^{d} \times \mathbb{R}^{d}}$ in (1.8). One fixes $(x, y) \in \mathbb{R}^{d} \times \mathbb{R}^{d}$, and decomposes its kernel, namely the function $g: \xi \mapsto K\left(x,\left\{H_{n}(y)\right\}, \xi\right)$ in the basis $\left\{\widehat{\psi}_{l, j, k}(-\xi)\right\}_{l, j, k}$. Then we apply the integral $\int_{\mathbb{R}} g(\cdot) d W$ to this decomposition. Since, this integral is an isometry from the Hilbert space $L^{2}\left(\mathbb{R}^{d}\right)$ into the Hilbert space $L^{2}(\Omega)$ of square integrable, mean-zero random variables, we obtain in view of (1.8)

$$
Y(x, y)=\sum_{l=1}^{2^{d}-1} \sum_{j \in \mathbb{Z}} \sum_{k \in \mathbb{Z}^{d}} a_{l, j, k}\left(x,\left\{H_{n}(y)\right\}\right) \epsilon_{l, j, k},
$$

where $\left\{\epsilon_{l, j, k}\right\}_{l, j, k}$ is a sequence of independent $\mathcal{N}(0,1)$ Gaussian random variables and where the non-random coefficients $a_{l, j, k}\left(x,\left\{H_{n}(y)\right\}\right)$ are given by

$$
\begin{aligned}
a_{l, j, k}\left(x,\left\{H_{n}(y)\right\}\right) & =\int_{\mathbb{R}^{d}} K\left(x,\left\{H_{n}(y)\right\}, \xi\right) \overline{\widehat{\psi}_{l, j, k}(-\xi)} d \xi \\
& =\int_{\mathbb{R}^{d}} K\left(x,\left\{H_{n}(y)\right\}, \xi\right) \widehat{\psi}_{l, j, k}(\xi) d \xi .
\end{aligned}
$$

sing techniques similar to those of the Proof of Proposition 2.2 of [6], one can show that the series (2.5) is with probability 1 , uniformly convergent for all $(x, y) \in K \times \mathbb{R}^{d}$, where $K$ is an arbitrary compact subset of $\mathbb{R}^{d}$. The Generalized Multifractional Field with Random Exponent (GMFRE) will be obtained by substituting to the deterministic functions $\left\{H_{n}\right\}$ in $(2.5)$ admissible stochastic processes $\left\{S_{n}\right\}$.

Definition 2.1 A sequence $\left(\left\{S_{n}(t)\right\}_{t \in \mathbb{R}^{d}}\right)_{n \in \mathbb{N}}$ of stochastic processes is admissible if for almost all $\omega$, it satisfies the following conditions:

(i) $S_{0}(., \omega)$ takes values in $(-\infty, 1)$ and for all $n \geq 1, S_{n}(., \omega)$ takes values in

$$
\left[\frac{1}{\log (64+n)}, 1-\frac{1}{\log (64+n)}\right]
$$

(ii) $S_{n}(., \omega)$ is a Lipschitz function, more precisely, a $C(n, \omega)$-Lipschitz function (that is, $\left|S_{n}(t, \omega)-S_{n}\left(t^{\prime}, \omega\right)\right| \leq C(n, \omega)\left|t-t^{\prime}\right|$ for all $\left.t, t^{\prime} \in \mathbb{R}\right)$.

(iii) The Lipschitz constants $C_{n}(\omega)$ depend on $n$ and increase slower than $n$, that is, $\lim _{n \rightarrow \infty} n^{-1} C_{n}(\omega)=0$.

(iv) For all integers $q \geq 1, S_{3 q}(\cdot, \omega)=S_{3 q+1}(\cdot, \omega)=S_{3 q+2}(\cdot, \omega)$. See Remark 3.1 for the motivation behind this technical condition.

$S_{0}$ will be related to the small frequency behavior of the process and will not play an important role, while the range of $S_{n}, n \geq 1$, increases with $n$ and tends to $(0,1)$ as $n \rightarrow \infty$. 
We are now in position to define the GMFRE and the GMPRE.

Definition 2.2 The GMFRE with parameter $\left(S_{n}\right)_{n \in \mathbb{N}}$ is the continuous field defined for almost all $\omega$ and each $(x, y) \in \mathbb{R}^{d} \times \mathbb{R}^{d}$ as the random series

$$
V(x, y, \omega)=\sum_{l=1}^{2^{d}-1} \sum_{j \in \mathbb{Z}} \sum_{k \in \mathbb{Z}^{d}} a_{l, j, k}\left(x,\left\{S_{n}(y, \omega)\right\}\right) \epsilon_{l, j, k}(\omega),
$$

where

- $\left\{\epsilon_{l, j, k}\right\}$ is a sequence of $\mathcal{N}(0,1)$ independent Gaussian random variables

- $\left(S_{n}\right)_{n \in \mathbb{N}}$ is admissible in the sense of Definition 2.1.

- for almost all $\omega$ and every $1 \leq l \leq 2^{d}-1, j \in \mathbb{Z}, k \in \mathbb{Z}^{d}$ and $(x, y) \in \mathbb{R}^{d} \times \mathbb{R}^{d}$,

$$
a_{l, j, k}\left(x,\left\{S_{n}(y, \omega)\right\}\right)=\int_{\mathbb{R}^{d}}\left[\sum_{n=0}^{\infty} \frac{\left(e^{i x . \xi}-1\right)}{|\xi|^{S_{n}(y, \omega)+d / 2}} \hat{f}_{n-1}(\xi)\right] \widehat{\psi}_{l, j, k}(\xi) d \xi
$$

- $\left\{\widehat{f}_{n-1}(\cdot)\right\}_{n \in \mathbb{N}}$ is a sequence of functions of the Schwartz class $S\left(\mathbb{R}^{d}\right)$, with values in $[0,1]$, that satisfy for every $\xi=\left(\xi_{1}, \ldots, \xi_{d}\right) \in \mathbb{R}^{d}$,

$$
\begin{aligned}
\widehat{f}_{-1}(\xi)= & \left\{\begin{array}{l}
1 \text { if }\left|\xi_{i}\right| \leq 2 \pi / 3, \text { for all } i=1, \ldots d, \\
0 \text { if }\left|\xi_{i}\right| \geq 4 \pi / 3, \text { for some } i
\end{array}\right. \\
& \widehat{f}_{0}(\xi)=\widehat{f}_{-1}\left(2^{-1} \xi\right)-\widehat{f}_{-1}(\xi),
\end{aligned}
$$

and, more generally, for all $n \in \mathbb{N}$,

$$
\widehat{f}_{n}(\xi)=\widehat{f}_{-1}\left(2^{-n-1} \xi\right)-\widehat{f}_{-1}\left(2^{-n} \xi\right)=\widehat{f}_{0}\left(2^{-n} \xi\right) .
$$

Remark 2.1 As in the proofs of Part (b) of Proposition 3.3 and Lemma 4.2, one can show that the series in (2.8) is, with probability 1 , uniformly convergent in $(x, y)$ on every compact subset of $\mathbb{R}^{d} \times \mathbb{R}^{d}$. This is why the definition of GMFRE makes sense.

Observe that the functions $\widehat{f}_{n}$ are compactly supported and if $D_{n}, n \in \mathbb{N}$, denotes the compact cube defined in (2.4), then one has

$$
\operatorname{supp} \widehat{f}_{-1} \subset D_{1}
$$

and for every $n \in \mathbb{N}$,

$$
\operatorname{supp} \widehat{f_{n}} \subset D_{n+2} \backslash D_{n} \text {. }
$$


Since the support of $\widehat{f}_{n}$ moves towards the high frequency range as $n$ increases, it is $S_{n}$ with large $n$ which determines the high frequency behavior of the process $V(\cdot, y)$. Observe finally that

$$
\sum_{n=0}^{\infty} \widehat{f}_{n-1}(\xi)=1
$$

Definition 2.3 The GMPRE with parameter $\left(S_{n}\right)_{n \in \mathbb{N}}$ is the continuous process $\{Z(t)\}_{t \in \mathbb{R}^{d}}$ defined for almost all $\omega$ and all $t \in \mathbb{R}^{d}$ as

$$
Z(t, \omega)=V(t, t, \omega) .
$$

The conditions (i) to (iv) in Definition 2.1 are not restrictive because:

Proposition 2.1 Let $\{S(t)\}_{t \in \mathbb{R}^{d}}$ be a stochastic process taking values in $[0,1]$ that satisfies almost surely, for all $t \in \mathbb{R}^{d}$,

$$
S(t)=\liminf _{n \rightarrow \infty} \widetilde{S}_{n}(t)
$$

where $\left(\left\{\widetilde{S}_{n}(t)\right\}_{t \in \mathbb{R}^{d}}\right)_{n \in \mathbb{N}}$ is a sequence of continuous processes. Then there exists an admissible sequence $\left(\left\{S_{n}(t)\right\}_{t \in \mathbb{R}^{d}}\right)_{n \in \mathbb{N}}$ which satisfies, almost surely for all $t \in \mathbb{R}^{d}$,

$$
S(t)=\liminf _{n \rightarrow \infty} S_{n}(t)
$$

Proof of Proposition 2.1. First, replacing $\widetilde{S}_{0}(t)$ by $\min \left(\frac{1}{2}, \widetilde{S}_{0}(t)\right)$ and $\widetilde{S}_{n}(t)$ with $n \geq 1$ by

$$
\max \left(\frac{2}{\log (64+n)}, \min \left(\widetilde{S}_{n}(t), 1-\frac{2}{\log (64+n)}\right)\right),
$$

one may suppose that for every $t \in \mathbb{R}^{d}$,

$$
\widetilde{S}_{0}(t) \in\left(-\infty, \frac{1}{2}\right]
$$

and

$$
\widetilde{S}_{n}(t) \in\left[\frac{2}{\log (64+n)}, 1-\frac{2}{\log (64+n)}\right] .
$$

Thus the sequence $\left(\left\{\widetilde{S}_{n}(t)\right\}_{t \in \mathbb{R}^{d}}\right)_{n \in \mathbb{N}}$ satisfies condition (i). Next we are going to construct a new sequence having the same lim inf as $\left(\left\{\widetilde{S}_{n}(t)\right\}_{t \in \mathbb{R}^{d}}\right)_{n \in \mathbb{N}}$ and satisfying conditions (i)-(iv). First, observe that, using Stone-Weierstrass 
approximation theorem, one can show that any process $\{Q(t)\}_{t \in \mathbb{R}^{d}}$ with continuous paths has the following property. For every compact cube $K \subset \mathbb{R}^{d}$ and for every $\epsilon>0$, there is a stochastic process $\left\{Q_{K, \epsilon}(t)\right\}_{t \in \mathbb{R}^{d}}$ with polynomial paths (depending on $K$ and $\epsilon$ ) such that

$$
\sup _{t \in K}\left|Q(t)-Q_{K, \epsilon}(t)\right| \leq \epsilon .
$$

The measurability of $Q_{K, \epsilon}$ follows from the fact that for each $t, Q_{K, \epsilon}(t)$ can be expressed as a linear combination of random variables $Q(s)$. For example, when $K=[0,1]^{d}$, one may choose

$$
Q_{K, \epsilon}(t)=\sum_{k_{1}, \ldots, k_{d}=0}^{p}\left(\prod_{i=1}^{d}\left(\begin{array}{c}
p \\
k_{i}
\end{array}\right) t_{i}^{k}\left(1-t_{i}\right)^{p-k_{i}}\right) Q\left(\frac{k_{1}}{p}, \ldots, \frac{k_{p}}{p}\right) .
$$

In this case, for all $\omega, Q_{K, \epsilon}(., \omega)$ is called the $p$ th Bernstein polynomial of the function $Q(., \omega)$.

Now, taking in (2.19), for every $n \in \mathbb{N}, Q(t)=\widetilde{S}_{n}(t), K=[-n-1, n+1]^{d}$ and $\epsilon=\frac{1}{2 \log (64+n)}$, it follows that there is $\left(\left\{\widetilde{S}^{I}(t)\right\}_{t \in \mathbb{R}^{d}}\right)_{n \in \mathbb{N}}$ a sequence of processes with polynomial paths such that

$$
\sup _{t \in K_{n}}\left|\widetilde{S}_{n}(t)-\widetilde{S}^{I}(t)\right| \leq \frac{1}{2 \log (2+n)}
$$

Now let $\theta: \mathbb{R} \rightarrow[0,1]$ be a compactly supported deterministic $C^{\infty}$ function, which satisfies

$$
\theta(x)=\left\{\begin{array}{l}
1 \text { if }|x| \leq 1 \\
0 \text { if }|x| \geq 3 / 2
\end{array}\right.
$$

and for every $n \in \mathbb{N}$ and $t \in \mathbb{R}^{d}$, let us set

$$
\widetilde{S}_{n}^{I I}(t)=\widetilde{S}_{n}^{I}(t) \theta\left(\frac{\|t\|^{2}}{(n+1)^{2}}\right)+\frac{1}{2 \log (64+n)} .
$$

It follows from (2.17) and (2.18) that the process $\left\{\widetilde{S}_{n}^{I I}(t)\right\}_{t \in \mathbb{R}^{d}}$ verifies (i). It also verifies (ii). Indeed, its paths are $C(n)$-Lipschitz functions, since they are $C^{\infty}$ functions and such that

$$
\sup \left\{\left|\frac{\partial \widetilde{S}_{n}^{I I}}{\partial t_{i}}(t)\right| ; t \in \mathbb{R}^{d}, i=1, \ldots, d\right\}<\infty .
$$

Observe that this inequality follows from (2.22) and (2.23). Now we are going to construct a sequence $\left\{\widetilde{S}_{n}^{I I I}(t)\right\}_{t \in \mathbb{R}^{d}}$ that has the same liminf as $\left\{\widetilde{S}_{n}(t)\right\}_{t \in \mathbb{R}^{d}}$ and satisfies (i), (ii) and (iii). 
To do so we need to introduce the sequence of random variables $\left(\tau_{n}\right)_{n \in \mathbb{N}}$ defined by induction as $\tau_{0}=0$ and for every integer $n \geq 1$, as

$$
\tau_{n}=\left\{\begin{array}{l}
\tau_{n-1} \text { if } C\left(\tau_{n-1}+1\right)>\sqrt{n} \\
\tau_{n-1}+1 \text { if } C\left(\tau_{n-1}+1\right) \leq \sqrt{n}
\end{array}\right.
$$

Then, for every $n \in \mathbb{N}$ and $t \in \mathbb{R}^{d}$, we set

$$
\widetilde{S}_{n}^{I I I}(t)=\widetilde{S}_{\tau_{n}}^{I I}(t) .
$$

Clearly, $\widetilde{S}_{0}^{I I I}(t) \in(0,1)$ and for every $n \geq 1$,

$$
\widetilde{S}_{n}^{I I I}(t) \in\left[\frac{1}{\log (64+n)}, 1-\frac{1}{\log (64+n)}\right] .
$$

It is also clear that the paths of the $\left\{\widetilde{S}_{n}^{I I I}(t)\right\}_{t \in \mathbb{R}^{d}}$ are $C\left(\tau_{n}\right)$-Lipschitz functions. So, all we need to prove is that, for almost all $\omega$,

$$
\lim _{n \rightarrow \infty} n^{-1} C\left(\tau_{n}\right)=0
$$

and that for every $t \in \mathbb{R}^{d}$,

$$
\liminf _{n \rightarrow \infty} \widetilde{S}_{n}^{I I I}(t, \omega)=\liminf _{n \rightarrow \infty} \widetilde{S}_{n}(t, \omega) .
$$

Relation (2.25) will result from the following inequality. For every $n \in \mathbb{N}$ and for almost all $\omega$,

$$
C\left(\tau_{n}(\omega)\right) \leq A(\omega) \sqrt{1+n},
$$

where $A=1+C(0)$. To prove this last inequality, we will argue by induction. It holds when $n=0$, so suppose that for some integer $n \geq 1$ and almost all $\omega$,

$$
C\left(\tau_{n-1}(\omega)\right) \leq A \sqrt{n}
$$

and let us show that $(2.27)$ holds for almost all $\omega$. We need to consider two cases: $C\left(\tau_{n-1}(\omega)+1\right) \leq \sqrt{n}$ and $C\left(\tau_{n-1}(\omega)+1\right)>\sqrt{n}$.

When $C\left(\tau_{n-1}(\omega)+1\right) \leq \sqrt{n}$, it follows from $(2.24)$ that $\tau_{n}(\omega)=\tau_{n-1}(\omega)+1$. Therefore, we have that

$$
C\left(\tau_{n}(\omega)\right)=C\left(\tau_{n-1}(\omega)+1\right) \leq \sqrt{n} \leq A(\omega) \sqrt{1+n} .
$$

When $C\left(\tau_{n-1}(\omega)+1\right)>\sqrt{n}$, it follows from $(2.24)$ that $\tau_{n}(\omega)=\tau_{n-1}(\omega)$. Then, using the induction hypothesis, namely the inequality (2.28), one obtains that

$$
C\left(\tau_{n}(\omega)\right)=C\left(\tau_{n-1}(\omega)\right) \leq A(\omega) \sqrt{n} \leq A(\omega) \sqrt{n+1} .
$$


To show that Relation (2.26) holds for almost all $\omega$ and every $t \in \mathbb{R}^{d}$, it is sufficient to show that the sequence $\left\{\tau_{n}(\omega)\right\}_{n \in \mathbb{N}}$ takes all the integer values, that is for every $k \in \mathbb{N}$, there is $n \in \mathbb{N}$ such that $\tau_{n}(\omega)=k$. We will argue by induction on $k$. When $k=0$, set $n=0$. Suppose that $k \geq 1$ and there is $n_{1} \in \mathbb{N}$, such that $\tau_{n_{1}}(\omega)=k-1$ and let us prove that there exists $n_{2} \in \mathbb{N}$ for which $\tau_{n_{2}}(\omega)=k$. Set

$$
n_{2}=\min \left\{n \in \mathbb{N} ; n>n_{1} \text { and } C\left(\tau_{n_{1}}(\omega)\right) \leq \sqrt{n}\right\}
$$

which is always possible because $n_{1}$ is fixed and $\sqrt{n}$ increases with $n$. Define finally for all $t \in \mathbb{R}^{d}$, the sequence $\left(\left\{S_{n}(t)\right\}_{t \in \mathbb{R}^{d}}\right)_{n \in \mathbb{N}}$ as $S_{0}(t)=\widetilde{S}_{0}^{I I I}(t)$, $S_{1}(t)=\widetilde{S}_{1}^{I I I}(t), S_{2}(t)=\widetilde{S}_{2}^{I I I}(t)$ and for all integer $q \geq 1$ as $S_{3 q}(t)=$ $S_{3 q+1}(t)=S_{3 q+2}(t)=\widetilde{S}_{q}^{I I I}(t)$. It clearly satisfies (i), (ii), (iii) and (iv) and has the same lim inf as $\left\{\widetilde{S}_{n}^{I I I}(t)\right\}$ and hence as $\left\{\widetilde{S}_{n}(t)\right\}$.

\section{The low and high frequency components}

We now turn to the proof of Theorem 1.2 about GMFREs. The idea is to fix $t_{0} \in \mathbb{R}^{d}$ and to set

$$
W(t):=V\left(t, t_{0}\right)=R(t)+T(t), t \in \mathbb{R}^{d},
$$

where $\{R(t)\}_{t \in \mathbb{R}^{d}}$ and $\{T(t)\}_{t \in \mathbb{R}^{d}}$ are respectively the low frequency and the high frequency components of $\{W(t)\}_{t \in \mathbb{R}^{d}}$ (see Proposition 3.2). We analyze the pointwise Hölder exponent of $R$ in this section and that of $T$ in the next section.

We need some preliminary results. As indicated in Section 2, we use the Lemarié-Meyer wavelet basis of the space $L^{2}\left(\mathbb{R}^{d}\right)$ (see [29]) which is of the form:

$$
\left\{2^{j d / 2} \psi_{l}\left(2^{j} x-k\right) ; 1 \leq l \leq 2^{d}-1, j \in \mathbb{Z}, k \in \mathbb{Z}^{d}\right\}
$$

with $\psi_{l} \in \mathcal{S}\left(\mathbb{R}^{d}\right)$. To simplify the notations, set for every $j \in \mathbb{N}$,

$$
s_{j}=S_{j}\left(t_{0}\right) \quad \text { and } \quad \mu_{j}=\min \left(s_{j}, s_{j+1}, s_{j+2}\right) .
$$

In view of Part (iv) of Definition 2.1, one has

$$
\liminf _{j \rightarrow \infty} s_{j}=\liminf _{j \rightarrow \infty} \mu_{j}=\liminf _{q \rightarrow \infty} \mu_{3 q} .
$$

We will need the following definitions. 
Definition 3.1 For every integers $j \geq 1$ and $1 \leq l \leq 2^{d}-1$ let $\psi_{s, l}^{j}$ and $\widetilde{\psi}_{s, l}^{j}$ be the real-valued functions of the Schwartz class $S\left(\mathbb{R}^{d}\right)$ whose Fourier transforms are defined, for every $\xi \in \mathbb{R}^{d}$, by

$$
\widehat{\psi_{s, l}^{j}}(\xi)=\left(\sum_{p=0}^{2} 2^{-j\left(s_{j+p}-\mu_{j}\right)} \frac{\hat{f}_{0}\left(2^{1-p} \xi\right)}{|\xi|^{s_{j+p}+d / 2}}\right) \widehat{\psi}_{l}(\xi)
$$

and

$$
\widehat{\bar{\psi}_{s, l}^{j}}(\xi)=\left(\sum_{p=0}^{2} 2^{-j\left(s_{j+p}-\mu_{j}\right)} \frac{\hat{f}_{0}\left(2^{1-p} \xi\right)}{|\xi|^{s_{j+p}+d / 2}}\right)^{-1} \widehat{\psi}_{l}(\xi) .
$$

Definition 3.2 Two families of functions $\left(e_{n}\right)_{n \in \mathbb{N}}$ and $\left(f_{n}\right)_{n \in \mathbb{N}}$ are biorthogonal systems in $L^{2}\left(\mathbb{R}^{d}\right)$ if for any $n \in \mathbb{N}$ and $m \in \mathbb{N}$

$$
\int_{\mathbb{R}^{d}} e_{n}(x) \overline{f_{m}(x)} d x=\delta_{n, m} .
$$

Remark 3.1 Observe that in view of Part (iv) of Definition 2.1 one has, for all integers $q \geq 1$ and $1 \leq l \leq 2^{d}-1$ and every $\xi \in \mathbb{R}^{d}$,

$$
\widehat{\psi_{s, l}^{3 q}}(\xi)=\frac{\widehat{\psi_{l}}(\xi)}{|\xi|^{s_{3 q}+d / 2}} \quad \text { and } \quad \widehat{\bar{\psi}_{s, l}^{3 q}}(\xi)=\mid \xi^{s_{3 q}+d / 2} \widehat{\psi}_{l}(\xi) \text {. }
$$

Proposition 3.1 The functions $\psi_{s, l}^{j}$ and $\widetilde{\psi}_{s, l}^{j}$ have the following properties:

(a) The systems

$$
\left\{2^{j d / 2} \psi_{s, l}^{j}\left(2^{j} t-k\right) ; j \geq 1,1 \leq l \leq 2^{d}-1, k \in \mathbb{Z}^{d}\right\}
$$

and

$$
\left\{2^{j d / 2} \widetilde{\psi}_{s, l}^{j}\left(2^{j} t-k\right) ; j \geq 1,1 \leq l \leq 2^{d}-1, k \in \mathbb{Z}^{d}\right\}
$$

are biorthogonal.

(b) The functions $\psi_{s, l}^{j}$ belong to the Schwartz class uniformly in $j$, i.e. $\forall \alpha \in \mathbb{N}^{d}, \forall L \geq 0, \exists a>0$, one has

$$
\begin{gathered}
\forall j \geq 1,1 \leq l \leq 2^{d}-1, x=\left(x_{1}, \ldots, x_{d}\right) \in \mathbb{R}^{d}, \\
\left|\partial^{\alpha} \psi_{s, l}^{j}(x)\right| \leq a\left[\prod_{r=1}^{d}\left(2+\left|x_{r}\right|\right)\right]^{-L} .
\end{gathered}
$$

(c) The functions $\widetilde{\psi}_{s, l}^{3 q}$ belong to the Schwartz class uniformly in $j$, i.e. $\forall \alpha \in \mathbb{N}^{d}, \forall L \geq 0, \exists b>0$, one has

$$
\begin{gathered}
\forall j=3 q, q \geq 1,1 \leq l \leq 2^{d}-1, x \in \mathbb{R}^{d}, \\
\left|\partial^{\alpha} \widetilde{\psi}_{s, l}^{3 q}(x)\right| \leq b\left[\prod_{r=1}^{d}\left(2+\left|x_{r}\right|\right)\right]^{-L} .
\end{gathered}
$$


Proof of Proposition 3.1. We first prove Part (a). It follows from Parseval formula that

$$
\begin{gathered}
I\left(l, j, k ; l^{\prime}, j^{\prime}, k^{\prime}\right)=2^{\left(j+j^{\prime}\right) d / 2} \int_{\mathbb{R}^{d}} \psi_{s, l}^{j}\left(2^{j} t-k\right) \overline{\widetilde{\psi}_{s, l^{\prime}}^{j^{\prime}}\left(2^{j^{\prime}} t-k^{\prime}\right)} d t \\
=2^{-\left(j+j^{\prime}\right) d / 2} \int_{\mathbb{R}^{d}} e^{-i\left(2^{-j} k-2^{-j^{\prime}} k^{\prime}\right) \cdot \xi \widehat{\psi}_{s, l}^{j}\left(2^{-j} \xi\right) \widehat{\widetilde{\bar{\psi}_{s, l^{\prime}}^{j^{\prime}}}}\left(2^{-j^{\prime}} \xi\right)} d \xi .
\end{gathered}
$$

This last integral vanishes when $j^{\prime} \notin\{j-1, j, j+1\}$ since (3.5), (3.6) and (2.3) imply that the Lebesgue measure of the set $\operatorname{supp} \widehat{\psi}_{s, l}^{j}\left(2^{-j}\right.$.) $\cap$ $\operatorname{supp} \widehat{\psi}_{s, l^{\prime}}^{j^{\prime}}\left(2^{-j} \cdot\right)$ is equal to zero.

We will show next that $I\left(l, j, k ; l^{\prime}, j, k^{\prime}\right)=\delta\left(l, j, k ; l^{\prime}, j, k^{\prime}\right)$. Using (3.5), (3.6), (3.9) and the orthonormality of the functions $2^{j d / 2} \psi_{l}\left(2^{j} t-k\right)$ and $2^{j d / 2} \psi_{l^{\prime}}\left(2^{j} t-k\right)$ we obtain that

$$
\begin{aligned}
I\left(l, j, k ; l^{\prime}, j, k^{\prime}\right) & =2^{-j d} \int_{\mathbb{R}^{d}} e^{-i\left(k-k^{\prime}\right) \cdot\left(2^{-j} \xi\right)} \widehat{\psi}_{s, l}^{j}\left(2^{-j} \xi\right) \overline{\widehat{\vec{\psi}}_{s, l^{\prime}}^{j}\left(2^{-j} \xi\right)} d \xi \\
& =2^{-j d} \int_{\mathbb{R}^{d}} e^{-i\left(k-k^{\prime}\right) \cdot\left(2^{-j} \xi\right)} \widehat{\psi}_{l}\left(2^{-j} \xi\right) \overline{\widehat{\psi}_{l^{\prime}}\left(2^{-j} \xi\right)} d \xi \\
& =2^{j d} \int_{\mathbb{R}^{d}} \psi_{l}\left(2^{j} t-k\right) \overline{\psi_{l^{\prime}}\left(2^{j} t-k^{\prime}\right)} d t \\
& =\delta\left(l, j, k ; l^{\prime}, j, k^{\prime}\right) .
\end{aligned}
$$

To conclude the proof of Part (a), it is sufficient to show that $I\left(l, j, k ; l^{\prime}, j+\right.$ $\left.1, k^{\prime}\right)=0$ since one can get $I\left(l, j, k ; l^{\prime}, j-1, k^{\prime}\right)=0$ in the same way. It follows from (2.3) that

$$
\operatorname{supp} \widehat{\psi}_{l, s}^{j}\left(2^{-j} .\right) \cap \operatorname{supp} \widehat{\psi}_{l, s}^{j+1}\left(2^{-j-1} .\right) \subset D_{j+2} \backslash D_{j+1} .
$$

Since by (2.14), supp $\widehat{f}_{0}\left(2^{-n} \cdot\right) \subset D_{n+2} \backslash D_{n}$, we get up to a multiplicative constant that we neglect,

$$
\begin{aligned}
& I\left(l, j, k ; l^{\prime}, j+1, k^{\prime}\right) \\
& =\int_{D_{j+2} \backslash D_{j+1}}\left(2^{-j\left(s_{j+1}-\mu_{j}\right)} \frac{\widehat{f_{0}}\left(2^{-j} \xi\right)}{\left|2^{-j} \xi\right|^{s_{j+1}+d / 2}}+2^{-j\left(s_{j+2}-\mu_{j}\right)} \frac{\widehat{f}_{0}\left(2^{-j-1} \xi\right)}{\left|2^{-j} \xi\right|^{s_{j+2}+d / 2}}\right) \\
& \cdot\left(2^{-(j+1)\left(s_{j+1}-\mu_{j+1}\right)} \frac{\widehat{f_{0}}\left(2^{-j} \xi\right)}{\left|2^{-j-1} \xi\right|^{s_{j+1}+d / 2}}+2^{-(j+1)\left(s_{j+2}-\mu_{j+1}\right)} \frac{\widehat{f}_{0}\left(2^{-j-1} \xi\right)}{\left|2^{-j-1} \xi\right|^{s_{j+2}+d / 2}}\right)^{-1} \\
& \cdot e^{-i\left(2^{-j} k-2^{-j-1} k^{\prime}\right) \cdot \xi} \widehat{\psi}_{l}\left(2^{-j} \xi\right) \overline{\widehat{\widetilde{\psi}}_{l^{\prime}}\left(2^{-j-1} \xi\right)} d \xi \\
& =\int_{\mathbb{R}^{d}} e^{-i\left(2^{-j} k-2^{-j-1} k^{\prime}\right) \cdot \xi} \widehat{\psi}_{l}\left(2^{-j} \xi\right) \overline{\widehat{\psi}_{l^{\prime}}\left(2^{-j-1} \xi\right)} d \xi \\
& =\int_{\mathbb{R}^{d}} \psi_{l}\left(2^{j} t-k\right) \overline{\psi_{l^{\prime}}\left(2^{j+1} t-k^{\prime}\right)} d t=0 .
\end{aligned}
$$


We now prove Part (b). We will only show that the inequality (3.8) holds since the inequality (3.7) can be obtained in the same way. One has, up to the multiplicative factor $(2 \pi)^{-d / 2}, \widetilde{\psi}_{l, s}^{3 q}(x)=\int_{\mathbb{R}^{d}} e^{i x \cdot \xi} \widehat{\widetilde{\psi}}_{l, s}^{3 q}(\xi) d \xi$, and hence $\partial^{\alpha} \widetilde{\psi}(x)=i^{|\alpha|} \int_{\mathbb{R}^{d}} e^{i x \cdot \xi} \xi_{1}^{\alpha_{1}} \xi_{2}^{\alpha_{2}} \ldots \xi_{d}^{\alpha_{d}} \widehat{\widetilde{\psi}}_{l, s}(\xi) d \xi$, where $|\alpha|=\sum_{r=1}^{d} \alpha_{r}$. Set $e^{i \delta \cdot \xi} \widehat{\widetilde{\phi}^{3 q, \alpha}}(\xi)=\xi_{1}^{\alpha_{1}} \xi_{2}^{\alpha_{2}} \ldots \xi_{d}^{\alpha_{d}} \widehat{\widetilde{\psi}}_{l, s}(\xi)$, where the multi-index $\delta=\left(\delta_{1}, \ldots \delta_{d}\right)$ is such that for every $i, \delta_{i}=2$ when $x_{i} \geq 0$ and $\delta_{i}=-2$ otherwise. Observe that $\delta_{i}+x_{i}$ equals $2+\left|x_{i}\right|$ if $x_{i} \geq 0$ and equals $-\left(2+\left|x_{i}\right|\right)$ if $x_{i}<0$.

Integrating $L d$ times by parts we obtain

$$
\begin{aligned}
\left|\partial^{\alpha} \widetilde{\psi}_{l, s}^{3 q}(x)\right| & =\left|\int_{\mathbb{R}^{d}} e^{i(\delta+x) \cdot \xi} \widehat{\widetilde{\phi}^{3 q, \alpha}}(\xi) d \xi\right| \\
& =\left(\prod_{r=1}^{d}\left(2+\left|x_{r}\right|\right)\right)^{-L}\left|\int_{\mathbb{R}^{d}} e^{i(\delta+x) \cdot \xi} \partial^{\lambda} \widehat{\widetilde{\phi}^{3 q, \alpha}}(\xi) d \xi\right|
\end{aligned}
$$

where $\lambda$ is the multi-index whose components are equal to $L$. Let us now bound $\left|\partial^{\lambda} \widehat{\widetilde{\phi}^{3 q, \alpha}}(\xi)\right|$ when $\xi \in D_{2} \backslash D_{0}$. Relations (3.3), (3.6), (2.15) and Part (iv) of Definition 2.1 imply that $\widehat{\widetilde{\psi}_{l, s}^{3 q}}(\xi)=|\xi|^{s_{3 q}+d / 2} \widehat{\psi}_{l}(\xi)$, and hence $e^{i \delta \cdot \xi} \widehat{\widetilde{\phi}^{3 q, \alpha}}(\xi)=\xi_{1}^{\alpha_{1}} \xi_{2}^{\alpha_{2}} \ldots \xi_{d}^{\alpha_{d}}|\xi|^{s_{3 q}+d / 2} \widehat{\psi}_{l}(\xi)$. Applying Leibniz formula one can show that the function $\left|\partial^{\lambda} \widehat{\widetilde{\phi}^{3 q, \alpha}}(\xi)\right|$ is bounded by a linear combination of functions of the form $\left|\xi_{1}^{\mu_{1}} \xi_{2}^{\mu_{2}} \ldots \xi_{d}^{\mu_{d}}\right||\xi|^{s_{3 q}+d / 2-p}\left|\partial^{\nu} \widehat{\psi}_{l}(\xi)\right|$, where $\left(\mu_{1}, \ldots, \mu_{d}\right) \in$ $\mathbb{N}^{d}, \nu \in \mathbb{N}^{d}$ and $p \in \mathbb{N}$. Moreover, $\widehat{\psi}_{l}$ has support on $D_{2} \backslash D_{0}$ (see (2.3)) and (2.4) implies that for every $\xi \in D_{2} \backslash D_{0}$, one has $|\xi|>1$ and

$$
\begin{aligned}
& \left|\xi_{1}^{\mu_{1}} \ldots \xi_{d}^{\mu_{d}}\right||\xi|^{s_{3 q}+d / 2-p}\left|\partial^{\nu} \widehat{\psi}_{l}(\xi)\right| \\
& \quad \leq\left(\frac{8 \pi}{3}\right)^{|\mu|}\left(\frac{8 \pi}{3} \sqrt{d}\right)^{s_{3 q}+d / 2} \sup _{\xi \in D_{2}}\left|\partial^{\nu} \widehat{\psi}_{l}(\xi)\right| \leq c_{1},
\end{aligned}
$$

where $c_{1}>0$ is a constant that does not depend on $q$ and where we used (2.7). Finally (3.10) and (3.11) entail that for every integer $q \geq 1$ and $x \in \mathbb{R}^{d}$,

$$
\begin{aligned}
\left|\partial^{\alpha} \widetilde{\psi}^{3 q}(x)\right| & \leq \prod_{r=1}^{d}\left(2+\left|x_{r}\right|\right)^{-L} \int_{D_{2} \backslash D_{0}}\left|e^{i \delta \cdot \xi} \partial^{\lambda} \widehat{\widetilde{\phi}^{3 q, \alpha}}(\xi)\right| d \xi \\
& \leq c_{2} \prod_{r=1}^{d}\left(2+\left|x_{r}\right|\right)^{-L}
\end{aligned}
$$

where $c_{2}>0$ is a constant that does not depend on $q$. 
The next result provides a wavelet decomposition of the process $\{W(t)\}$. Whereas usually wavelets are independent of the scale $j \in \mathbb{Z}$, here they depend on $j$. When $j \geq 1$, the wavelets are $\psi_{s, l}^{j}$ (defined in (3.5)); when $j=0$ and $j=-1$, they are $\psi_{s, l}^{0}$ and $\psi_{s, l}^{-1}$ (defined in (3.12) and (3.13)); when $j \leq-2$, we have a single wavelet $\psi_{s, l}^{-2}$ (defined in (3.14)). Note that in the decomposition (3.17) below we change the sign of the indices, so that $\sum_{j=-\infty}^{0}$ becomes $\sum_{j=0}^{\infty}$.

Definition 3.3 Let $\psi_{s, l}^{0}, \psi_{s, l}^{-1}, \psi_{s, l}^{-2}$ be the functions of $S\left(\mathbb{R}^{d}\right)$ defined for every $x \in \mathbb{R}^{d}$ as,

$$
\begin{aligned}
\psi_{s, l}^{0}(x) & =\int_{\mathbb{R}^{d}} e^{i x \cdot \xi}\left(\frac{\widehat{f}_{-1}(\xi)}{|\xi|^{s_{0}+d / 2}}+\frac{\widehat{f}_{0}(\xi)}{|\xi|^{s_{1}+d / 2}}+\frac{\widehat{f}_{1}(\xi)}{|\xi|^{s_{2}+d / 2}}\right) \widehat{\psi}_{l}(\xi) d \xi \\
\psi_{s, l}^{-1}(x) & =\int_{\mathbb{R}^{d}} e^{i x \xi} \frac{\left(\widehat{f}_{-1}\left(2^{-1} \xi\right)+\widehat{f}_{0}\left(2^{-1} \xi\right)\right) \widehat{\psi}_{l}(\xi)}{|\xi|^{s_{0}+d / 2}} d \eta
\end{aligned}
$$

and

$$
\psi_{s, l}^{-2}(x)=\int_{\mathbb{R}^{d}} e^{i x \xi} \frac{\widehat{\psi}_{l}(\xi)}{|\xi|^{s_{0}+d / 2}} d \xi
$$

Proposition 3.2 For every $t \in \mathbb{R}^{d}$, one has

$$
W(t)=R(t)+T(t)
$$

where

$$
T(t)=\sum_{l=1}^{2^{d}-1} \sum_{j=1}^{\infty} \sum_{k \in \mathbb{Z}^{d}} 2^{-j \mu_{j}} \psi_{s, l}^{j}\left(2^{j} t-k\right) \epsilon_{l, j, k}
$$

and, with the convention that $m(0)=0, m(1)=1$ and for all $j \geq 2, m(j)=2$,

$$
\begin{aligned}
R(t)= & \sum_{l=1}^{2^{d}-1} \sum_{j=0}^{\infty} \sum_{k \in \mathbb{Z}^{d}} 2^{j s_{0}}\left(\psi_{s, l}^{-m(j)}\left(2^{-j} t-k\right)-\psi_{s, l}^{-m(j)}(-k)\right) \epsilon_{l,-j, k} \\
& +\sum_{l=1}^{2^{d}-1} \sum_{j=1}^{\infty} \sum_{k \in \mathbb{Z}^{d}} 2^{-j \mu_{j}} \psi_{s, l}^{j}(-k) \epsilon_{l,-j, k} .
\end{aligned}
$$

Here $\left\{\epsilon_{l, j, k}\right\}$ is a sequence of independent $\mathcal{N}(0,1)$ Gaussian random variables, $\psi_{s, l}^{j}$ with $j \geq 1$, the functions introduced in (3.5). 
The processes $\{R(t)\}_{t \in \mathbb{R}^{d}}$ and $\{T(t)\}_{t \in \mathbb{R}^{d}}$ can be viewed respectively as the low frequency and the high frequency components of $\{W(t)\}_{t \in \mathbb{R}^{d}}$.

Proposition 3.3 The processes $\{R(t)\}_{t \in \mathbb{R}^{d}}$ and $\{T(t)\}_{t \in \mathbb{R}^{d}}$ have the following properties:

(a) With probability 1 , the paths of $\{R(t)\}_{t \in \mathbb{R}^{d}}$ are $C^{\infty}$ functions.

(b) With probability 1, the series (3.16) and (3.17) are uniformly convergent in $t$, on every compact subset of $\mathbb{R}^{d}$.

(c) For all $l, j, k$ one has

$$
2^{j d} \int_{\mathbb{R}^{d}} T(t) \widetilde{\psi}_{s, l}^{j}\left(2^{j} t-k\right) d t=2^{-j \mu_{j}} \epsilon_{l, j, k}
$$

We will use the following lemma of [5] in the proof of Proposition 3.2.

Lemma 3.1 Let $\left\{\epsilon_{M} ; M=\left(m_{1}, \ldots, m_{d}\right) \in \mathbb{Z}^{d}\right\}$ be a sequence of $\mathcal{N}(0,1)$ Gaussian random variables (not necessarily independent). Then there exist random variables $C, C^{\prime}>0$ with finite moments of any order such that, almost surely for all $M \in \mathbb{Z}^{d}$,

$$
\left|\epsilon_{M}\right| \leq C \sqrt{\log \left(2+\sum_{k=1}^{d}\left|m_{k}\right|\right)} \leq C^{\prime} \sqrt{\prod_{k=1}^{n} \log \left(2+\left|m_{k}\right|\right)}
$$

Proof of Proposition 3.2. Let us prove (3.15), (3.16) and (3.17). It follows from (2.8), (3.1) and (3.3) that the process $\{W(t)\}_{t \in \mathbb{R}^{d}}$ can be represented as the random series

$$
W(t, \omega)=\sum_{l=1}^{2^{d}-1} \sum_{j \in \mathbb{Z}} \sum_{k \in \mathbb{Z}^{d}} a_{l, j, k}\left(t,\left\{s_{n}(\omega)\right\}\right) \epsilon_{l, j, k}(\omega),
$$

which is, as we will show, a.s. uniformly convergent in $t$ on every compact subset of $\mathbb{R}^{d}$. We first want to determine the random coefficients $a_{l, j, k}\left(t,\left\{s_{n}\right\}\right)$. Using the inclusions,

- $\operatorname{supp} \widehat{f}_{-1} \subset D_{1}$,

- for all $n \geq 0, \operatorname{supp} \widehat{f}_{n} \subset D_{n+2} \backslash D_{n}$,

- for all $j \in \mathbb{Z}, k \in \mathbb{Z}^{d}$ and $1 \leq l \leq 2^{d}-1$, supp $\widehat{\psi}_{l, j, k} \subset D_{j+2} \backslash D_{j}$, 
we obtain that for every $k \in \mathbb{Z}^{d}$ and $1 \leq l \leq 2^{d}-1$,

$$
a_{l,-j, k}\left(t,\left\{s_{n}\right\}\right)=\int_{\mathbb{R}^{d}} \frac{e^{i t \cdot \xi}-1}{|\xi|^{s_{0}+d / 2}}\left(\widehat{f}_{-1}(\xi)+\widehat{f}_{0}(\xi)\right) \widehat{\psi}_{l,-j, k}(\xi) d \xi
$$

if $j \leq-1$,

$$
\begin{aligned}
& a_{l, 0, k}\left(t,\left\{s_{n}\right\}\right)= \\
& =\int_{\mathbb{R}^{d}}\left(e^{i t \cdot \xi}-1\right)\left(\frac{\widehat{f}_{-1}(\xi)}{|\xi|^{s_{0}+d / 2}}+\frac{\widehat{f_{0}}(\xi)}{|\xi|^{s_{1}+d / 2}}+\frac{\widehat{f}_{0}\left(2^{-1} \xi\right)}{|\xi|^{s_{2}+d / 2}}\right) \widehat{\psi}_{l, 0, k}(\xi) d \xi
\end{aligned}
$$

if $j=0$, and

$$
a_{l, j, k}\left(t,\left\{s_{n}\right\}\right)=\int_{\mathbb{R}^{d}}\left(e^{i t \cdot \xi}-1\right)\left(\sum_{p=0}^{2} \frac{\widehat{f_{0}}\left(2^{-j+1-p} \xi\right)}{|\xi|^{s_{j+p}+d / 2}}\right) \widehat{\psi}_{l, j, k}(\xi) d \xi
$$

if $j \geq 1$.

Next, by setting $\eta=2^{j} \xi$ in (3.21) and using the equality $\widehat{\psi}_{l, j, k}(\xi)=$ $2^{-j d / 2} e^{-i k .\left(2^{-j} \xi\right)} \widehat{\psi}_{l}\left(2^{-j} \xi\right),(3.22),(2.3)$, we get

$$
a_{l,-j, k}\left(t,\left\{s_{n}\right\}\right)=2^{j s_{0}}\left(\psi_{s, l}^{-m(j)}\left(2^{-j} t-k\right)-\psi_{s, l}^{-m(j)}(-k)\right) .
$$

Similarly, (3.23) yields

$$
a_{l, j, k}\left(t,\left\{s_{n}\right\}\right)=2^{-j \mu_{j}}\left(\psi_{s, l}^{j}\left(2^{j} t-k\right)-\psi_{s, l}^{j}(-k)\right),
$$

where $\psi_{s, l}^{j}$ is defined in (3.5).

Proof of Proposition 3.3. Let us prove (a). It is sufficient to show that the series (3.17) and the series

$$
\sum_{l=1}^{2^{d}-1} \sum_{j=0}^{\infty} \sum_{k \in \mathbb{Z}^{d}} 2^{j\left(s_{0}-|\alpha|\right)} \partial^{\alpha} \psi_{s, l}^{-m(j)}\left(2^{-j} t-k\right),
$$

where the multi-index $\alpha \neq 0$ is arbitrary, are with probability 1 , uniformly convergent in $t$ on every compact subset $B$ of $\mathbb{R}^{d}$. There is no restriction to suppose that $B=[0,1]^{d}$. Since the functions $\psi_{s, l}^{-m(j)}, j \in \mathbb{N}$ belong to $S\left(\mathbb{R}^{d}\right)$, for every multi-index $\beta \in \mathbb{N}^{d}$ and real $L>0$, there is a constant $c_{1}>0$, such that the inequality

$$
\left|\partial^{\beta} \psi_{s, l}^{-m(j)}(x)\right| \leq c_{1}\left(\prod_{r=1}^{d}\left(2+\left|x_{r}\right|\right)\right)^{-L},
$$

holds for every $x=\left(x_{1}, \ldots, x_{d}\right) \in \mathbb{R}^{d}$ and $j \in \mathbb{N}$. Observe that the constant $c_{1}$ does not depend on $j$, since the range of $m(j)$ is finite. 
Applying the Mean Value Theorem one gets that for any integer $j \geq 1$, $t \in B$ and $k \in \mathbb{Z}^{d}$, there is a real $\theta \in(0,1)$ such that

$$
\psi_{s, l}^{-m(j)}\left(2^{-j} t-k\right)-\psi_{s, l}^{-m(j)}(-k)=2^{-j} \sum_{r=1}^{d} \frac{\partial \psi_{s, l}^{-m(j)}}{\partial x_{r}}\left(\theta 2^{-j} t-k\right) t_{r} .
$$

Using this last equality and (3.27) one obtains that

$$
\begin{aligned}
\left|\psi_{s, l}^{-m(j)}\left(2^{-j} t-k\right)-\psi_{s, l}^{-m(j)}(-k)\right| & \leq c_{1} d 2^{-j}\left(\prod_{r=0}^{d}\left(2+\left|\theta 2^{-j} t_{r}-k_{r}\right|\right)\right)^{-L} \\
& \leq c_{2} 2^{-j} \prod_{r=1}^{d}\left(1+\left|k_{r}\right|\right)^{-L}
\end{aligned}
$$

where $c_{2}>0$ is a constant (which does not depend on $j$ ). It then follows from Part (i) of Definition 2.1, Lemma 3.1 and (3.28), that there is a random variable $C_{3}>0$ such that with probability 1 , for all $t \in B$,

$$
\begin{aligned}
|R(t)| \leq & C_{3} \sum_{j=0}^{\infty} \sum_{k \in \mathbb{Z}^{d}} 2^{-j\left(1-s_{0}\right)} \log ^{1 / 2}(2+j) \prod_{r=1}^{d} \frac{\log ^{1 / 2}\left(2+\left|k_{r}\right|\right)}{\left(1+\left|k_{r}\right|\right)^{L}} \\
& +C_{3} \sum_{j=0}^{\infty} \sum_{k \in \mathbb{Z}^{d}} 2^{-j / \log (64+j)} \log ^{1 / 2}(2+j) \prod_{r=1}^{d} \frac{\log ^{1 / 2}\left(2+\left|k_{r}\right|\right)}{\left(1+\left|k_{r}\right|\right)^{L}} \\
<\infty &
\end{aligned}
$$

by choosing $L$ sufficiently large, which proves that, with probability 1 , the series (3.17) is uniformly convergent in $t \in B$. One can prove similarly that the series (3.26) is with probability 1 , uniformly convergent in $t \in B$.

To prove (b), note that the uniform convergence of $R(t)$ follows from the proof of (a) and the uniform convergence of $T(t)$ is established in the proof of Lemma 4.2.

(c) is a straightforward consequence of the biorthogonality condition and (3.16). Observe that the existence of the integral in (3.18) is assured by the biorthogonality condition and Lemma 4.2 below.

Observe that (a) of Proposition 3.3 implies

Corollary 3.1 Let $\left\{\alpha_{W}(t)\right\}_{t \in \mathbb{R}^{d}}$ and $\left\{\alpha_{T}(t)\right\}_{t \in \mathbb{R}^{d}}$ be respectively the pointwise Hölder exponent of the processes $\{W(t)\}_{t \in \mathbb{R}^{d}}$ and $\{T(t)\}_{t \in \mathbb{R}^{d}}$ defined in (3.1) and in (3.16). Then almost surely for all $t \in \mathbb{R}^{d}$, one has

$$
\alpha_{W}(t)=\alpha_{T}(t) .
$$




\section{Analysis of the high frequency component $T(t)$}

In view of (3.29), we need now to determine the pointwise Hölder exponent at $t_{0}$ of the process $\{T(t)\}_{t \in \mathbb{R}^{d}}$. defined in (3.16). We first provide an upper bound to this exponent.

Proposition 4.1 There exists $\Omega_{1}^{*}$, an event of probability 1 , which does not depend on $t_{0}$ and such that the relations

$$
\alpha_{T}\left(t_{0}, \omega\right) \leq \liminf _{j \rightarrow \infty} s_{j}(\omega)
$$

and

$$
\limsup _{t \rightarrow t_{0}} \frac{\left|T(t, \omega)-T\left(t_{0}, \omega\right)\right|}{\left|t-t_{0}\right|}=\infty
$$

holds for all $\omega \in \Omega_{1}^{*}$.

To prove Proposition 4.1, we need the following lemma which shows that after rescaling the indices of a sequence of independent Gaussian random variables one can always find a large component whose index is "close" to any $t_{0} \in \mathbb{R}$.

Lemma 4.1 Let $\left\{\epsilon_{l, j, k}\right\}$ be a sequence of independent $\mathcal{N}(0,1)$ Gaussian random variables. There is $\Omega_{1}^{*}$, an event of probability 1 , which is independent of $t_{0} \in \mathbb{R}$ and satisfies the following property. For all $\omega \in \Omega_{1}^{*}$ and every integers $1 \leq l \leq 2^{d}-1$ and $j$ big enough, there is $\widehat{k}_{j} \in \mathbb{Z}^{d}$ such that

$$
\left|t_{0}-2^{-j} \widehat{k}_{j}\right| \leq c j 2^{-j}
$$

where $c>0$ is a constant (independent of $\omega$ ) and

$$
\left|\epsilon_{l, j, \widehat{k}_{j}}(\omega)\right| \geq 1 / 4
$$

Proof of Lemma 4.1. Given $t_{0} \in \mathbb{R}^{d}$ there is $m_{0} \in \mathbb{N}$ such that $t_{0} \in$ $\left[-m_{0}, m_{0}\right]^{d}$. Consider the set of all dyadic numbers of order $j$ in $\left(-m_{0}, m_{0}\right)^{d}$, namely the set $\left\{2^{-j} k ; k \in \Delta_{j}\right\}$ where

$$
\Delta_{j}=\left\{-m_{0}\left(2^{j}-1\right), \ldots, m_{0}\left(2^{j}-1\right)\right\}^{d} .
$$

Clearly, there is $\widetilde{k}_{j} \in \Delta_{j}$ such that

$$
\left|t_{0}-2^{-j} \widetilde{k}_{j}\right| \leq \sqrt{d} 2^{-j}
$$

We shall now introduce sets $D_{m_{0}, j}^{q}$ and $I_{m_{0}, j}$ such that, by letting

$$
\Delta_{j} \subset \bigcup_{q \in I_{m_{0}, j}} D_{m_{0}, j}^{q}
$$

we will have

$$
\widetilde{k}_{j} \in D_{m_{0}, j}^{\widetilde{q}_{j}} \text { for some } \widetilde{q}_{j} \in I_{m_{0}, j} \text {. }
$$


Heuristically, if $d=1, D_{m_{0}, j}^{q}$ are those numbers in $\Delta_{j}$ whose ratio is $q \in \mathbb{Z}$ when divided by $j$ (i.e $k=q j+r, r$ denoting the remainder). We let $I_{m_{0}, j}$ be the set of all such ratios $q$. To gain some flexibility, we relax these heuristic definitions and use the following formal definitions of $I_{m, j}$ and $D_{m, j}^{q}$. For every integers $m \geq 1$ and $j \geq 1$, we let

$$
I_{m, j}=\left\{-\left[\frac{m 2^{j}}{j}\right],-\left[\frac{m 2^{j}}{j}\right]+1, \ldots,\left[\frac{m 2^{j}}{j}\right]-1,\left[\frac{m 2^{j}}{j}\right]\right\}^{d}
$$

and for every $q \in I_{m, j}$, we let

$$
D_{m, j}^{q}=\left\{j q+\sum_{i=1}^{d} n_{i} e_{i} ; \forall i, n_{i} \in\{-(j-1), \ldots, j-1\}\right\}
$$

where $e_{i}$ is the element of $\mathbb{Z}^{d}$ whose $i$-th component equals 1 and the other components equal $0\left(\sum_{i=1}^{d} n_{i} e_{i}\right.$ denotes the "remainder"). Now fix the in$\operatorname{dex} l$ in $\left\{\epsilon_{l, j, k}\right\}$. We want to show that the events

$$
A_{m, j}=\bigcup_{q \in I_{m, j}} \bigcap_{k \in D_{m, j}^{q}}\left(\left|\epsilon_{l, j, k}\right|<1 / 4\right)
$$

satisfy

$$
\sum_{j=1}^{\infty} P\left(A_{m, j}\right)<\infty
$$

Since the $\epsilon_{l, j, k}$ are independent $\mathcal{N}(0,1)$ Gaussian random variables, it follows that for all $q$,

$$
P\left(\bigcap_{k \in D_{m, j}^{q}}\left(\left|\epsilon_{l, j, k}\right|<1 / 4\right)\right)=\prod_{k \in D_{m, j}^{q}} P\left(\left|\epsilon_{l, j, k}\right|<1 / 4\right) \leq(2 \sqrt{2 \pi})^{-(2 j-1)^{d}} .
$$

Hence

$$
P\left(A_{m, j}\right) \leq \sum_{q \in I_{m, j}} P\left(\bigcap_{k \in D_{m, j}^{q}}\left(\left|\epsilon_{l, j, k}\right|<1 / 4\right)\right) \leq\left(\frac{m 2^{j+1}}{j}+1\right)^{d}(2 \sqrt{2 \pi})^{-(2 j-1)^{d}}
$$

and (4.10) results from this last inequality. The Borel-Cantelli lemma implies that for every integer $m \geq 1$,

$$
P\left(\bigcup_{J=1}^{\infty} \bigcap_{j=J}^{\infty} A_{m, j}^{c}\right)=1
$$

where $A_{m, j}^{c}$ denotes the complementary of the event $A_{m, j}$, namely the event

$$
A_{m, j}^{c}=\bigcap_{q \in I_{m, j}} \bigcup_{k \in D_{m, j}^{q}}\left(\left|\epsilon_{l, j, k}\right| \geq 1 / 4\right) .
$$


Now consider

$$
\Omega_{1}^{*}=\bigcap_{m \geq 1} \bigcup_{J \geq 1} \bigcap_{j \geq J} A_{m, j}^{c}
$$

an event with probability 1. Fix $\omega \in \Omega_{1}^{*}$. There is an $m=m_{0}$ and an integer $j$ big enough, so that $\left|\epsilon_{l, j, \widehat{k}_{j}}(\omega)\right| \geq 1 / 4$, where $\widehat{k}_{j} \in D_{m_{0}, j}^{\widetilde{q}_{j}}$ by (4.6). Relations (4.8) and (4.5) imply that

$\left|t_{0}-2^{-j} \widehat{k}_{j}\right| \leq\left|t_{0}-2^{-j} \widetilde{k}_{j}\right|+2^{-j}\left|\widetilde{k}_{j}-\widehat{k}_{j}\right| \leq \sqrt{d} 2^{-j}+\sqrt{d} 2^{-j}(2 j-1) \leq c j 2^{-j}$, which completes the proof.

The following lemma controls how fast the paths of the process $\{T(t)\}_{t \in \mathbb{R}^{d}}$ increase as $t \rightarrow \infty$.

Lemma 4.2 There is a random variable $C>0$ with finite moments of any order such that almost surely for all $t \in \mathbb{R}^{d}$,

$$
|T(t)| \leq C \log ^{1 / 2}(2+|t|) .
$$

Proof of Lemma 4.2. If $x=\left(x_{1}, \ldots, x_{d}\right)$ is a vector of $\mathbb{R}^{d}$, then $[x]$ will denote the vector whose components are the integer parts of the component of $x$ i.e $[x]=\left(\left[x_{1}\right], \ldots,\left[x_{d}\right]\right)$. It follows from Relations (3.3), (3.7), (3.16) and (3.19), from Part (i) of Definition 2.1, from the sub-additivity of the function $y \mapsto \log ^{1 / 2}(2+y)$ and from the inequality $\sqrt{a+b} \leq \sqrt{a}+\sqrt{b}$, that almost surely for all $t \in \mathbb{R}^{d}$,

$$
\begin{aligned}
|T(t)| \leq & \sum_{l=1}^{2^{d}-1} \sum_{j=1}^{\infty} \sum_{k \in \mathbb{Z}^{d}} 2^{-j \mu_{j}}\left|\epsilon_{l, j, k}\right|\left|\psi_{s, l}^{j}\left(2^{j} t-k\right)\right| \\
\leq & C_{1} \sum_{j=1}^{\infty} \sum_{k \in \mathbb{Z}^{d}} \frac{2^{-j / \log (64+j)} \log ^{1 / 2}(2+j+|k|)}{\left(2+\left|2^{j} t-k\right|\right)^{d+1}} \\
= & C_{1} \sum_{j=1}^{\infty} 2^{-j / \log (64+j)} \sum_{k \in \mathbb{Z}^{d}} \frac{\log ^{1 / 2}\left(2+j+\mid k+\left[2^{j} t\right]\right)}{\left(2+\left|2^{j} t-\left[2^{j} t\right]-k\right|\right)^{d+1}} \\
\leq & C_{2} \sum_{j=1}^{\infty} 2^{-j / \log (64+j)} \log ^{1 / 2}(2+j)+C_{2} \sum_{j=1}^{\infty} 2^{-j / \log (64+j)} \log ^{1 / 2}\left(2+2^{j}\right) \\
& +C_{2}\left(\sum_{j=1}^{\infty} 2^{-j / \log (64+j)}\right) \log ^{1 / 2}(2+|t|)
\end{aligned}
$$

where $C_{1}>0$ is a random variable with finite moments of any order and $C_{2}=C_{1} \sup _{x \in[0,1]^{d}}\left\{\sum_{k \in \mathbb{Z}^{d}} \frac{\log ^{1 / 2}(2+|k|)}{(2+|x-k|)^{d+1}}\right\}$. 
Lemma 4.3 For each $j=-2,-1,0,1, \ldots$ the moments of any order of the functions $\psi_{s, l}^{j}$ and $\widetilde{\psi}_{s, l}^{j}$ vanish, namely one has

$$
\int_{\mathbb{R}^{d}} t_{1}^{n_{1}} t_{2}^{n_{2}} \ldots t_{d}^{n_{d}} \psi_{s, l}^{j}(t) d t=\int_{\mathbb{R}^{d}} t_{1}^{n_{1}} t_{2}^{n_{2}} \ldots t_{d}^{n_{d}} \widetilde{\psi}_{s, l}^{j}(t) d t=0
$$

for all $\left(n_{1}, \ldots, n_{d}\right) \in \mathbb{N}^{d}$.

Proof of Lemma 4.3. One has up to a multiplicative factor

$$
\int_{\mathbb{R}^{d}} t_{1}^{n_{1}} t_{2}^{n_{2}} \ldots t_{d}^{n_{d}} \psi_{s, l}^{j}(t) d t=\partial^{\left(n_{1}, \ldots, n_{d}\right)} \widehat{\psi}_{s, l}^{j}(0)=0
$$

and this partial derivatives is equal to 0 since the function $\widehat{\psi}_{s, l}^{j}$ vanishes in the neighborhood of 0 . One can similarly show that $\partial^{\left(n_{1}, \ldots, n_{d}\right)}{\widetilde{\psi_{s, l}^{j}}}_{(0)}(0$.

Let us now introduce the pointwise Hölder spaces.

Definition 4.1 Let $f$ be a complex-valued, continuous and nowhere differentiable function defined on $\mathbb{R}^{d}, t_{0}$ be a point of $\mathbb{R}^{d}$ and $\alpha \in[0,1)$. One says that $f$ belongs to the pointwise Hölder space $C^{\alpha}\left(t_{0}\right)$ if there is a constant $c>0$ such that the inequality

$$
\left|f(t)-f\left(t_{0}\right)\right| \leq c\left|t-t_{0}\right|^{\alpha}
$$

holds for every $\left|t-t_{0}\right|$ small enough (the constant $c$ generally depends on the point $\left.t_{0}\right)$.

Remark 4.1 Because of the embedding

$$
C^{\alpha_{2}}\left(t_{0}\right) \subset C^{\alpha_{1}}\left(t_{0}\right), \quad 0 \leq \alpha_{1} \leq \alpha_{2}<1,
$$

the pointwise Hölder exponent $\alpha_{f}\left(t_{0}\right)$ of the function $f$ at $t_{0}$ can be expressed as

$$
\alpha_{f}\left(t_{0}\right)=\sup \left\{\alpha ; f \in C^{\alpha}\left(t_{0}\right)\right\}
$$

Now we can prove Proposition 4.1.

Proof of Proposition 4.1. Pick $\omega_{0} \in \Omega_{1}^{*}$ where $\Omega_{1}^{*}$ is the event of probability 1, introduced in Lemma 4.1. Assume that (4.2) is satisfied (we will show below that this is indeed the case). Then $\alpha_{T}\left(t_{0}, \omega_{0}\right) \leq 1$, which implies that (4.1) holds when $\liminf _{j \rightarrow \infty} s_{j}\left(\omega_{0}\right)=1$. Suppose that $\liminf _{j \rightarrow \infty} s_{j}\left(\omega_{0}\right)<1$. We shall assume ad absurdum that for some $\gamma_{0} \in$ $(0,1)$ satisfying

$$
\gamma_{0}>\liminf _{j \rightarrow \infty} s_{j}\left(\omega_{0}\right),
$$

the function $t \mapsto T(t, \omega)$ belongs to $C^{\gamma_{0}}\left(t_{0}\right)$. 
It follows from the definition of the space $C^{\gamma_{0}}\left(t_{0}\right)$, that there is a constant $c_{1}>0$ such that for any $\left|t-t_{0}\right|$ small enough,

$$
\left|T\left(t, \omega_{0}\right)-T\left(t_{0}, \omega_{0}\right)\right| \leq c_{1}\left|t-t_{0}\right|^{\gamma_{0}} .
$$

Because of (4.11), this inequality holds as well for any $t \in \mathbb{R}^{d}$. Then using (3.18), (4.12), (4.17) and (3.8) one obtains that for all integers $q \geq 1$ and $1 \leq l \leq 2^{d}-1$ and all $k \in \mathbb{Z}^{d}$,

$$
\begin{aligned}
2^{-3 q \mu_{3 q}}|| \epsilon_{l, 3 q, k}\left(\omega_{0}\right) \mid & =2^{3 q d}\left|\int_{\mathbb{R}^{d}} T\left(t, \omega_{0}\right) \widetilde{\psi}_{s, l}^{3 q}\left(2^{3 q} t-k\right) d t\right| \\
& =2^{3 q d}\left|\int_{\mathbb{R}^{d}}\left(T\left(t, \omega_{0}\right)-T\left(t_{0}, \omega_{0}\right)\right) \widetilde{\psi}_{s, l}^{3 q}\left(2^{3 q} t-k\right) d t\right| \\
& \leq 2^{3 q d} \int_{\mathbb{R}^{d}}\left|T\left(t, \omega_{0}\right)-T\left(t_{0}, \omega_{0}\right)\right|\left|\widetilde{\psi}_{s, l}^{3 q}\left(2^{3 q} t-k\right)\right| d t \\
& \leq c_{1} 2^{3 q d} b \int_{\mathbb{R}^{d}} \frac{\left|t-t_{0}\right|^{\gamma_{0}}}{\left(2+\left|2^{3 q} t-k\right|\right)^{L}} d t
\end{aligned}
$$

where the real $L$ has been chosen such that $L \geq \gamma_{0}+d$ to ensure that the last integral converges. Setting $u=2^{3 q} t-k$ in the last integral, one gets

$$
\begin{aligned}
2^{-3 q \mu_{3 q}}\left|\epsilon_{l, 3 q, k}\left(\omega_{0}\right)\right| & \leq c_{1} b \int_{\mathbb{R}^{d}} \frac{\left|2^{-3 q}(u+k)-t_{0}\right|^{\gamma_{0}}}{(2+|u|)^{L}} \\
& \leq c_{2} b\left(\int_{\mathbb{R}^{d}} \frac{\left|2^{-3 q} u\right|^{\gamma_{0}}}{(2+|u|)^{L}} d u+\left|2^{-3 q} k-t_{0}\right|^{\gamma_{0}} \int_{\mathbb{R}^{d}} \frac{d u}{(2+|u|)^{L}}\right) \\
& \leq c_{3} b 2^{-3 q \gamma_{0}}\left(1+\left|2^{3 q} t_{0}-k\right|\right)^{\gamma_{0}} .
\end{aligned}
$$

Now set in Relation (4.18) $k=\widehat{k}_{3 q}$ (where $\widehat{k}_{3 q}$ was introduced in Lemma 4.1). Relations (4.3) and (4.4) then imply that for all integer $q \geq 1$ and $n \geq 1$,

$$
\frac{2^{-3 q \mu_{3 q}}}{4} \leq c_{4} b_{q} 2^{-3 q \gamma_{0}}(1+q)^{\gamma_{0}} \leq c_{5} 2^{-3 q \gamma_{0}}(1+q)^{n+\gamma_{0}} .
$$

In view of (3.4), the inequalities (4.19) and (4.16) cannot both hold.

We prove finally that (4.2) is satisfied. Suppose ad absurdum that for some constant $c_{5}>0$ and all $\left|t-t_{0}\right|$ small enough,

$$
\left|T\left(t, \omega_{0}\right)-T\left(t_{0}, \omega\right)\right| \leq c_{5}\left|t-t_{0}\right|
$$

Then, applying the same methods as before, one can show that (4.20) and the relation $s_{j}\left(\omega_{0}\right) \in\left[\frac{1}{\log (64+j)}, 1-\frac{1}{\log (64+j)}\right], j \geq 1$, cannot both hold. 
Our goal now is to show that $\lim _{\inf }{ }_{j \rightarrow \infty} s_{j}$ is a lower bound to the pointwise Hölder exponent of the process $\{T(t)\}_{t \in \mathbb{R}^{d}}$ at $t_{0}$. In fact, we will show more generally, that this quantity is a lower bound to the uniform Hölder regularity of $\{T(t)\}_{t \in \mathbb{R}^{d}}$. We first recall the definition of the uniform Hölder spaces and some elementary results about them.

Definition 4.2 Let $U$ be a nonempty open subset of $\mathbb{R}^{d}$ and $\gamma \in(0,1)$. One says that a complex valued function $f$ defined over $U$ belongs to the uniform Hölder space $C^{\gamma}(U)$ if and only if $f$ satisfies the following conditions: there is a constant $c>0$, such that the inequality

$$
\left|f\left(t^{\prime}\right)-f\left(t^{\prime \prime}\right)\right| \leq c\left|t^{\prime}-t^{\prime \prime}\right|^{\gamma}
$$

holds for all $t^{\prime} \in U$ and $t^{\prime \prime} \in U$.

Lemma 4.4 (a) Let $U$ be an open nonempty subset of $\mathbb{R}^{d}$, the inclusion

$$
C^{\gamma_{2}}(U) \subset C^{\gamma_{1}}(U)
$$

holds for all reals $0<\gamma_{1} \leq \gamma_{2}<1$.

(b) When a function $f$ belongs to $C^{\gamma}(U)$ then, for all $t_{0} \in U$, it belongs to the pointwise Hölder space $C^{\gamma}\left(t_{0}\right)$ and its pointwise Hölder exponent at $t_{0}$ is at least $\gamma$.

Proposition 4.2 There is $\Omega_{2}^{*}$, an event of probability 1 , which does not depend of $t_{0}$ and satisfies the following property. For any $\omega \in \Omega_{2}^{*}$ such that $\liminf _{j \rightarrow \infty} s_{j}(\omega)>0$ and for any real $\tau>0$, the function $t \mapsto T(t, \omega)$ belongs to the Hölder space $C^{\gamma}\left((-\tau, \tau)^{d}\right)$ when $\gamma \in\left(0, \liminf _{j \rightarrow \infty} s_{j}(\omega)\right)$. Therefore, the inequality

$$
\liminf _{j \rightarrow \infty} s_{j}(\omega) \leq \alpha_{T}\left(t_{0}, \omega\right),
$$

holds for any $\omega \in \Omega_{2}^{*}$.

Proof of Proposition 4.2. We will show that almost surely, for any $r \in$ $\left(0, \liminf _{j \rightarrow \infty} s_{j}(\omega)\right), \epsilon>$ small enough and $t^{\prime}, t^{\prime \prime} \in(-\tau, \tau)^{d}$,

$$
\left|T\left(t^{\prime}\right)-T\left(t^{\prime \prime}\right)\right| \leq C_{1}\left|t^{\prime}-t^{\prime \prime}\right|^{r-\epsilon},
$$

where $C_{1}>0$ is a random variable that only depends on $r$ and $\epsilon$.

As liminf $\operatorname{in}_{j \rightarrow \infty} \mu_{j}=\liminf _{j \rightarrow \infty} s_{j}>r$, there is an integer $j_{0}$ such that the inequality $\mu_{j}>\gamma$, holds for all $j \geq j_{0}+1$. Writing $\sum_{j=0}^{\infty}=\sum_{j=1}^{j_{0}}+\sum_{j_{0}+1}^{\infty}$ and majorizing $\sum_{j=j_{0}+1}^{\infty}$, one has that, for every $t^{\prime}, t^{\prime \prime} \in(-\tau, \tau)^{d}$,

$$
\left|T\left(t^{\prime}\right)-T\left(t^{\prime \prime}\right)\right| \leq G\left(t^{\prime}, t^{\prime \prime}\right)+L\left(t^{\prime}, t^{\prime \prime}\right)
$$


where

$$
\begin{aligned}
G\left(t^{\prime}, t^{\prime \prime}\right) & =\left|\sum_{l=1}^{2^{d}-1} \sum_{j=1}^{j_{0}} \sum_{k \in \mathbb{Z}^{d}} 2^{-j \mu_{j}} \epsilon_{l, j, k}\left(\psi_{s, l}^{j}\left(2^{j} t^{\prime}-k\right)-\psi_{s, l}^{j}\left(2^{j} t^{\prime \prime}-k\right)\right)\right| \\
L\left(t^{\prime}, t^{\prime \prime}\right) & =\sum_{l=1}^{2^{d}-1} \sum_{j=1}^{\infty} \sum_{k \in \mathbb{Z}^{d}} 2^{-j r}\left|\epsilon_{l, j, k}\right|\left|\psi_{s, l}^{j}\left(2^{j} t^{\prime}-k\right)-\psi_{s, l}^{j}\left(2^{j} t^{\prime \prime}-k\right)\right| .
\end{aligned}
$$

We first consider $G$ and show that there is a random variable $C_{3}>0$, such that almost surely for all $t^{\prime}, t^{\prime \prime} \in(-\tau, \tau)^{d}$,

$$
G\left(t^{\prime}, t^{\prime \prime}\right) \leq C_{3}\left|t^{\prime}-t^{\prime \prime}\right|
$$

In fact it is sufficient to show that with probability 1 , for all real $\rho>0$, all multi-index $\beta \in \mathbb{N}^{d}$ and all integers $j \geq 1$ and $1 \leq l \leq 2^{d}-1$, the series $\sum_{k \in \mathbb{Z}^{d}} \epsilon_{l, j, k} \partial^{\beta} \psi_{s, l}^{j}(x-k)$ is uniformly convergent in $x$, when $|x| \leq$ $\rho$. Indeed, this result implies that, with probability $1, x \mapsto g_{j, l}(x)=$ $\sum_{k \in \mathbb{Z}^{d}} \epsilon_{l, j, k} \partial^{\alpha} \psi_{s, l}^{j}(x-k)$ is a $C^{\infty}$ function over $(-\tau-1, \tau+1)^{d}$ and consequently a Lipschitz function over $[-\tau, \tau]^{d}$. It follows from (3.7) and (3.19), that there is a random variable $C_{2}>0$ (depending on $j$ ) such that, with probability 1 ,

$$
\begin{aligned}
\sum_{k \in \mathbb{Z}^{d}}\left|\epsilon_{l, j, k}\right|\left|\partial^{\beta} \psi_{s, l}^{j}(x-k)\right| & \leq C_{2} \sum_{k \in \mathbb{Z}^{d}} \frac{\log ^{1 / 2}(2+|k|)}{(1+\rho+|x-k|)^{d+1}} \\
& \leq C_{2} \sum_{k \in \mathbb{Z}^{d}} \frac{\log ^{1 / 2}(2+|k|)}{(1+|k|)^{d+1}}<\infty
\end{aligned}
$$

since $\rho+|x-k| \geq \rho+|k|-|x| \geq|k|$. The random variable $C_{2}$ depends on $l$ and $j$ but since these have a finite range in (4.26) we conclude that (4.28) holds. We now turn to $L$ and show that for any arbitrarily small real $\epsilon>0$, there is a random variable $C_{24}>0$ such that almost surely for all $t^{\prime}, t^{\prime \prime} \in(-\tau, \tau)^{d}$,

$$
L\left(t^{\prime}, t^{\prime \prime}\right) \leq C_{24}\left|t^{\prime}-t^{\prime \prime}\right|^{r-\epsilon} .
$$

There is no restriction to suppose that $0<\left|t^{\prime}-t^{\prime \prime}\right|<1 / 2$. Let $j_{1} \geq 1$ be the integer such that

$$
2^{-j_{1}-1} \leq\left|t^{\prime}-t^{\prime \prime}\right|<2^{-j_{1}}
$$

For all integers $j \geq 1$ and $1 \leq i \leq d$ let

$$
D_{j, i}=\left\{k=\left(k_{1}, \ldots, k_{d}\right) \in \mathbb{Z}^{d} ;\left|k_{i}\right| \geq 2^{j+1} \tau\right\}
$$

and let

$$
D_{j}=\bigcup_{i=1}^{d} D_{j, i} .
$$


$D_{j, i}^{c}$ and $D_{j}^{c}$ will respectively denote the complements of $D_{j, i}$ and $D_{j}$. One has

$$
L\left(t^{\prime}, t^{\prime \prime}\right)=\sum_{n=1}^{4} L_{n}\left(t^{\prime}, t^{\prime \prime}\right)
$$

where

$$
\begin{aligned}
& L_{1}\left(t^{\prime}, t^{\prime \prime}\right)=\sum_{l=1}^{2^{d}-1} \sum_{j=1}^{j_{1}} \sum_{k \in D_{j}} 2^{-j r}\left|\epsilon_{l, j, k}\right|\left|\psi_{s, l}^{j}\left(2^{j} t^{\prime}-k\right)-\psi_{s, l}^{j}\left(2^{j} t^{\prime \prime}-k\right)\right| \\
& L_{2}\left(t^{\prime}, t^{\prime \prime}\right)=\sum_{l=1}^{2^{d}-1} \sum_{j=1}^{j_{1}} \sum_{k \in D_{j}^{c}} 2^{-j r}\left|\epsilon_{l, j, k}\right|\left|\psi_{s, l}^{j}\left(2^{j} t^{\prime}-k\right)-\psi_{s, l}^{j}\left(2^{j} t^{\prime \prime}-k\right)\right|, \\
& L_{3}\left(t^{\prime}, t^{\prime \prime}\right)=\sum_{l=1}^{2^{d}-1} \sum_{j=j_{1}+1}^{\infty} \sum_{k \in D_{j}} 2^{-j r}\left|\epsilon_{l, j, k}\right|\left|\psi_{s, l}^{j}\left(2^{j} t^{\prime}-k\right)-\psi_{s, l}^{j}\left(2^{j} t^{\prime \prime}-k\right)\right| \\
& L_{4}\left(t^{\prime}, t^{\prime \prime}\right)= \\
& \sum_{l=1}^{2^{d}-1} \sum_{j=j_{1}+1}^{\infty} \sum_{k \in D_{j}^{c}} 2^{-j r}\left|\epsilon_{l, j, k}\right|\left|\psi_{s, l}^{j}\left(2^{j} t^{\prime}-k\right)-\psi_{s, l}^{j}\left(2^{j} t^{\prime \prime}-k\right)\right| .
\end{aligned}
$$

Let us bound $\sum_{k \in D_{j}}\left|\epsilon_{l, j, k}\right|\left|\psi_{s, l}^{j}\left(2^{j} t-k\right)\right|$ when $t \in(-\tau, \tau)^{d}$. It follows from (3.7) and (3.19) that there is $C_{4}>0$ a random variable such that almost surely for all $t \in(-\tau, \tau)^{d}$ and $j \geq 1$,

$$
\begin{aligned}
& \sum_{k \in D_{j}}\left|\epsilon_{l, j, k}\right|\left|\psi_{s, l}^{j}\left(2^{j} t-k\right)\right| \\
& \quad \leq C_{4} \log ^{1 / 2}(2+j) \sum_{i=1}^{d} \sum_{k \in D_{j, i}} \prod_{n=1}^{d}\left(\frac{\log ^{1 / 2}\left(2+\left|k_{n}\right|\right)}{\left(5+\left|2^{j} t_{n}-k_{n}\right|\right)^{L}}\right) .
\end{aligned}
$$

Moreover, for all integers $1 \leq i \leq d$ and $j \geq 1$, one has

$$
\begin{aligned}
& \sum_{k \in D_{j, i}} \prod_{n=1}^{d}\left(\frac{\log ^{1 / 2}\left(2+\left|k_{n}\right|\right)}{\left(5+\left|2^{j} t_{n}-k_{n}\right|\right)^{L}}\right) \\
& =\left(\sum_{\left|k_{i}\right| \geq 2^{j+1} \tau} \frac{\log ^{1 / 2}\left(2+\left|k_{i}\right|\right)}{\left(5+\left|2^{j} t_{i}-k_{i}\right|\right)^{L}}\right) \times \prod_{1 \leq n \leq d, n \neq i}\left(\sum_{k_{n} \in \mathbb{Z}} \frac{\log ^{1 / 2}\left(2+\left|k_{n}\right|\right)}{\left(5+\left|2^{j} t_{n}-k_{n}\right|\right)^{L}}\right)
\end{aligned}
$$

and for every $\left|t_{i}\right| \leq \tau$, one has

$$
\begin{aligned}
\sum_{\left|k_{i}\right| \geq 2^{j+1} \tau} \frac{\log ^{1 / 2}\left(2+\left|k_{i}\right|\right)}{\left(5+\left|2^{j} t_{i}-k_{i}\right|\right)^{L}} & \leq \sum_{\left|k_{i}\right| \geq 2^{j+1} \tau} \frac{\log ^{1 / 2}\left(2+\left|k_{i}\right|\right)}{\left(5+\left|k_{i}\right|-2^{j} \tau\right)^{L}} \\
& \leq 2 \sum_{k_{i}=\left[2^{j+1} \tau\right]}^{\infty} \frac{\log ^{1 / 2}\left(2+k_{i}\right)}{\left(4+k_{i}-\left[2^{j} \tau\right]\right)^{L}}
\end{aligned}
$$


Now set $m=k_{i}-\left[2^{j} \tau\right]$. When $k_{i} \geq\left[2^{j+1} \tau\right]$, one has $m \geq\left[2^{j+1} \tau\right]-\left[2^{j} \tau\right] \geq$ $2^{j+1} \tau-1-2^{j} \tau \geq 2^{j} \tau-1 \geq\left[2^{j} \tau\right]-2$. Thus,

$$
\begin{aligned}
\sum_{\left|k_{i}\right| \geq 2^{j+1} \tau} \frac{\log ^{1 / 2}\left(2+\left|k_{i}\right|\right)}{\left(5+\left|2^{j} t_{i}-k_{i}\right|\right)^{L}} & \leq 2 \sum_{m=\left[2^{j} \tau\right]-2}^{\infty} \frac{\log ^{1 / 2}\left(2+m+\left[2^{j} \tau\right]\right)}{(4+m)^{L}} \\
& \leq C_{5} \log ^{1 / 2}\left(2+2^{j} \tau\right) \sum_{m=\left[2^{j} \tau\right]-2}^{\infty} \frac{\log ^{1 / 2}(2+m)}{(4+m)^{L}} \\
& \leq C_{6} \log ^{1 / 2}\left(2+2^{j} \tau\right) \sum_{m=\left[2^{j} \tau\right]-2}(4+m)^{-L+\epsilon} \\
& \leq C_{6} \log ^{1 / 2}\left(2+2^{j} \tau\right) \int_{2^{j} \tau}^{+\infty}(1+x)^{-L+\epsilon} d x \\
& \leq C_{7} \log ^{1 / 2}\left(2+2^{j} \tau\right)\left(1+2^{j} \tau\right)^{-L+1+\epsilon},
\end{aligned}
$$

where one supposes that $L \geq 2$ and $\epsilon>0$ is arbitrarily small. Observe that the bound in (4.40) does not involve $i$ anymore. Hence it follows from (4.38), (4.39) and (4.40) that there is a random variable $C_{8}>0$, such that almost surely for all $t \in \mathbb{R}^{d}$ with $|t| \leq \tau$ and for all integers $j \geq 1$ and $1 \leq l \leq 2^{d}-1$, one has

$$
\begin{aligned}
& \sum_{k \in D_{j}}\left|\epsilon_{l, j, k}\right|\left|\psi_{s, l}^{j}\left(2^{j} t-k\right)\right| \\
& \quad \leq C_{8}\left(\log ^{1 / 2}(2+j) \log ^{1 / 2}\left(2+2^{j} \tau\right)\right)\left(1+2^{j} \tau\right)^{-L+1+\epsilon}
\end{aligned}
$$

Using this last inequality and (4.36) one obtains that

$$
\begin{aligned}
L_{3}\left(t^{\prime}, t^{\prime \prime}\right) & \leq \sum_{l=1}^{2^{d}-1} \sum_{j=j_{1}+1}^{\infty} \sum_{k \in D_{j}} 2^{-j r}\left|\epsilon_{l, j, k}\right|\left(\left|\psi_{s, l}^{j}\left(2^{j} t^{\prime}-k\right)\right|+\left|\psi_{s, l}^{j}\left(2^{j} t^{\prime \prime}-k\right)\right|\right) \\
& \leq C_{9} \sum_{j=j_{1}+1}^{\infty}\left(\log ^{1 / 2}(2+j) \log ^{1 / 2}\left(2+2^{j} \tau\right)\right) 2^{-j(r+L-1-\epsilon)} \\
& \leq C_{10} 2^{-\left(j_{1}+1\right)(r+L-1-2 \epsilon)} \\
& \leq C_{10}\left|t^{\prime}-t^{\prime \prime}\right|^{r+L-1-2 \epsilon},
\end{aligned}
$$

where, by (4.30), $C_{10}>0$ is a random variable which does not depend on $j_{1}$.

Let us now bound $L_{1}\left(t^{\prime}, t^{\prime \prime}\right)$. By the Mean Value Theorem, for all integer $j \geq 1$ and all $k \in \mathbb{Z}^{d}$, there is $\theta \in(0,1)$ (depending on $t^{\prime}, t^{\prime \prime}, j$ and $k$ ) such that

$$
\begin{aligned}
& \psi_{s, l}^{j}\left(2^{j} t^{\prime}-k\right)-\psi_{s, l}^{j}\left(2^{j} t^{\prime \prime}-k\right) \\
& \quad=2^{j} \sum_{r=1}^{d} \partial^{e_{r}} \psi_{s, l}^{j}\left(2^{j} t^{\prime}-k+\theta 2^{j}\left(t^{\prime \prime}-t^{\prime}\right)\right) \cdot\left(t_{r}^{\prime}-t_{r}^{\prime \prime}\right),
\end{aligned}
$$


where for all $r, e_{r} \in \mathbb{N}^{d}$ is the multi-index whose $r$-th component equals 1 and whose other components equal 0. Relation (3.7) implies that

$$
\begin{aligned}
& \left|\psi_{s, l}^{j}\left(2^{j} t^{\prime}-k\right)-\psi_{s, l}^{j}\left(2^{j} t^{\prime \prime}-k\right)\right| \\
& \quad \leq c_{11} 2^{j}\left|t^{\prime}-t^{\prime \prime}\right| \times \prod_{r=1}^{d}\left(2+\left|2^{j} t_{r}^{\prime}-k_{r}+2^{j} \theta\left(t_{r}^{\prime \prime}-t_{r}^{\prime}\right)\right|\right)^{-L}
\end{aligned}
$$

where $c_{11}>0$ is a constant depending on $j, k, t^{\prime}$ and $t^{\prime \prime}$. When $j \leq j_{1}$, using (4.30) one has that $2^{j}\left|t^{\prime}-t^{\prime \prime}\right| \leq 1$ and so it follows from (4.44) that

$$
\begin{aligned}
& \left|\psi_{s, l}^{j}\left(2^{j} t^{\prime}-k\right)-\psi_{s, l}^{j}\left(2^{j} t^{\prime \prime}-k\right)\right| \\
& \quad \leq c_{11} 2^{j}\left|t^{\prime}-t^{\prime \prime}\right| \times \prod_{r=1}^{d}\left(2+\left|2^{j} t_{r}^{\prime}-k_{r}\right|-2^{j}\left|t_{r}^{\prime \prime}-t_{r}^{\prime}\right|\right)^{-L} \\
& \quad \leq c_{11} 2^{j}\left|t^{\prime}-t^{\prime \prime}\right| \times \prod_{r=1}^{d}\left(1+\left|2^{j} t_{r}^{\prime}-k_{r}\right|\right)^{-L} .
\end{aligned}
$$

Relations (4.34), (3.19) and (4.45) imply that

$$
\begin{gathered}
L_{1}\left(t^{\prime}, t^{\prime \prime}\right) \\
(4.46) \leq c_{12} \sum_{j=1}^{j_{1}} \sum_{k \in D_{j}} 2^{-(r-1) j} \log ^{1 / 2}(2+j) \times \prod_{r=1}^{d}\left(\frac{\log ^{1 / 2}\left(2+\left|k_{r}\right|\right)}{\left(1+\left|2^{j} t_{r}^{\prime}-k_{r}\right|\right)^{L}}\right) \times\left|t^{\prime}-t^{\prime \prime}\right| .
\end{gathered}
$$

Using a method similar to the one that led to the inequality (4.41), one can show that for any arbitrary small real $\epsilon>0$ and all integer $j \geq 1$,

$$
\begin{aligned}
& \sum_{k \in D_{j}} 2^{-(r-1) j} \log ^{1 / 2}(2+j) \prod_{r=1}^{d}\left(\frac{\log ^{1 / 2}\left(2+\left|k_{r}\right|\right)}{\left(1+\left|2^{j} t_{r}^{\prime}-k_{r}\right|\right)^{L}}\right) \\
& \quad \leq c_{13} 2^{-(r-1) j} \log ^{1 / 2}(2+j) \log ^{1 / 2}\left(2+2^{j} \tau\right)\left(1+2^{j} \tau\right)^{-L+1+\epsilon} \\
& \quad \leq c_{14} 2^{-(r+L-2-2 \epsilon) j}
\end{aligned}
$$

where $c_{13}>0$ and $c_{14}>0$ are two constants that depend on $\epsilon$. It then follows from (4.46) and (4.47) that

$$
L_{1}\left(t^{\prime}, t^{\prime \prime}\right) \leq c_{15}\left(\sum_{j=1}^{j_{1}} 2^{-j(L+r-2-2 \epsilon)}\right)\left|t^{\prime}-t^{\prime \prime}\right| \leq c_{16}\left|t^{\prime}-t^{\prime \prime}\right|
$$

where $c_{16}=c_{15} \sum_{j=0}^{\infty} 2^{-j(L+r-2-2 \epsilon)}$. 
Let us now study $L_{4}\left(t^{\prime}, t^{\prime \prime}\right)$. It is convenient to bound

$$
\sum_{k \in D_{j}^{c}} 2^{-j r}\left|\epsilon_{l, j, k}\right|\left|\psi_{s, l}^{j}\left(2^{j} t-k\right)\right|
$$

when $t \in(-\tau, \tau)^{d}$.

Since on $D_{j}^{c}$ one has $\left|k_{i}\right|<2^{j+1} \tau$, Relations (3.7), (3.19), (4.31) and (4.32) imply that almost surely for all integer $j \geq 1$ and $t \in(-\tau, \tau)^{d}$,

$$
\begin{aligned}
\sum_{k \in D_{j}^{c}} 2^{-j r}\left|\epsilon_{l, j, k}\right|\left|\partial^{\alpha} \psi_{s, l}^{j}\left(2^{j} t-k\right)\right| & \leq C_{17} \sum_{k \in D_{j}^{c}} 2^{-j r} \frac{\log ^{1 / 2}(2+j+|k|)}{\left(2+\left|2^{j} t-k\right|\right)^{L}} \\
& \leq C_{17} \sum_{k \in \mathbb{Z}^{d}} 2^{-j r} \frac{\log ^{1 / 2}\left(2+j+2^{j+1} \tau d\right)}{\left(2+\left|2^{j} t-k\right|\right)^{L}} \\
& \leq C_{18} 2^{-j r} \log ^{1 / 2}\left(2+j+2^{j+1} \tau d\right),
\end{aligned}
$$

where $C_{17}>0$ is a random variable and

$$
C_{18}=C_{17} \sup _{x \in \mathbb{R}^{d}}\left\{\sum_{k \in \mathbb{Z}^{d}}(2+|x-k|)^{-L}\right\} .
$$

It follows from (4.37), (4.49) and (4.30) that

$$
L_{4}\left(t^{\prime}, t^{\prime \prime}\right) \leq C_{19} \sum_{j=j_{1}+1}^{\infty} 2^{-j(r-\epsilon)} \leq C_{19} 2^{-\left(j_{1}+1\right)(r-\epsilon)} \leq C_{19}\left|t^{\prime}-t^{\prime \prime}\right|^{r-\epsilon},
$$

where $\epsilon>0$ is arbitrarily small and $C_{19}>0$ is a random variable that only depends on $\epsilon$.

Let us now bound $L_{2}\left(t^{\prime}, t^{\prime \prime}\right)$. Relations (4.35), (4.45), (4.31) and (4.32) imply that

$$
\begin{aligned}
L_{2}\left(t^{\prime}, t^{\prime \prime}\right) & \leq C_{20}\left|t^{\prime}-t^{\prime \prime}\right| \sum_{j=1}^{j_{1}} \sum_{k \in D_{j}^{c}} 2^{-(r-1) j} \frac{\log ^{1 / 2}(2+j+|k|)}{\left.2+\left|2^{j} t-k\right|\right)^{L}} \\
& \leq C_{20}\left|t^{\prime}-t^{\prime \prime}\right| \sum_{j=1}^{j_{1}} \sum_{k \in D_{j}^{c}} \frac{2^{-(r-1) j} \log ^{1 / 2}\left(2+j+d 2^{j+1} \tau\right)}{\left(2+\left|2^{j} t-k\right|\right)^{L}} \\
& \leq C_{21}\left|t^{\prime}-t^{\prime \prime}\right| \sum_{j=1}^{j_{1}} 2^{-(r-1) j} \log ^{1 / 2}\left(2+j+d 2^{j+1} \tau\right)
\end{aligned}
$$

where $C_{20}>0$ is a random variable and

$$
C_{21}=C_{20} \sup _{x \in \mathbb{R}^{d}}\left\{\sum_{k \in \mathbb{Z}^{d}}(2+|x-k|)^{-L}\right\} .
$$


Finally, for all arbitrarily small $\epsilon>0$, there is a random variables $C_{22}>0$ and $C_{23}>0$, which only depends on $\epsilon$, such that

$$
L_{2}\left(t^{\prime}, t^{\prime \prime}\right) \leq C_{22}\left|t^{\prime}-t^{\prime \prime}\right| \sum_{j=0}^{j_{1}} 2^{-(r-1-\epsilon) j} \leq C_{23}\left|t^{\prime}-t^{\prime \prime}\right|^{r-\epsilon}
$$

where the last inequality follows from (4.30). Relations (4.25), (4.33), (4.42), (4.48), (4.50) and (4.51) imply the bound (4.24). This completes the proof.

Proof of Theorem 1.2. Corollary 3.1, Proposition 4.1 and Proposition 4.2 imply the theorem.

\section{Going from the GMFRE to the GMPRE}

The following proposition shows how a result on the pointwise Hölder exponent of the GMFRE implies a corresponding one on the GMPRE. We shall use the following lemma whose proof is similar to that of Proposition 1 of [5].

Lemma 5.1 Let $K$ be an arbitrary compact subset of $\mathbb{R}^{d}$. Then with probability $1, y \mapsto V(x, y, \omega)$ is a Lipschitz function over $K$. More precisely, there is a random variable $C>0$ of finite moments of any order, such that almost surely, for all $y, y^{\prime} \in K$, one has

$$
\sup _{x \in K}\left|V\left(x, y^{\prime}, \omega\right)-V\left(x, y^{\prime \prime}, \omega\right)\right| \leq C(\omega)\left|y^{\prime}-y^{\prime \prime}\right| .
$$

Proposition 5.1 Let $\{V(x, y)\}_{(x, y) \in \mathbb{R}^{d} \times \mathbb{R}^{d}}$ be a GMFRE and suppose that, almost surely for all $t_{0}$,

$$
\limsup _{t \rightarrow t_{0}} \frac{\left|V\left(t, t_{0}\right)-V\left(t_{0}, t_{0}\right)\right|}{\left|t-t_{0}\right|}=\infty
$$

and that the pointwise Hölder exponent at $t_{0}$ of the process $\left\{V\left(t, t_{0}\right)\right\}_{t \in \mathbb{R}^{d}}$ equals $\lim \inf _{n \rightarrow \infty} S_{n}\left(t_{0}\right)$. Then, almost surely for all $t_{0}$,

$$
\limsup _{t \rightarrow t_{0}} \frac{\left|Z(t)-Z\left(t_{0}\right)\right|}{\left|t-t_{0}\right|}=\infty
$$

and the pointwise Hölder exponent at $t_{0}$ of the GMPRE $\{Z(t)\}_{t \in \mathbb{R}^{d}}$ equals $\liminf \operatorname{in}_{n \rightarrow \infty} S_{n}\left(t_{0}\right)$.

Proof of Proposition 5.1. Let $\Omega^{*}$ be an event with probability 1 , such that for all $\omega \in \Omega^{*}$ and all $t_{0} \in \mathbb{R}^{d}$,

$$
\limsup _{t \rightarrow t_{0}} \frac{\left|V\left(t, t_{0}\right)-V\left(t_{0}, t_{0}\right)\right|}{\left|t-t_{0}\right|}=\infty,
$$

and the pointwise Hölder exponent at $t_{0}$ of the function $t \mapsto V\left(t, t_{0}, \omega\right)$ equals $\liminf \operatorname{in}_{n \rightarrow \infty} S_{n}\left(t_{0}, \omega\right)$. Fix $\omega \in \Omega^{*}$. 
We first prove that

$$
\limsup _{t \rightarrow t_{0}} \frac{\left|Z(t, \omega)-Z\left(t_{0}, \omega\right)\right|}{\left|t-t_{0}\right|}=\infty
$$

and

$$
\alpha_{Z}\left(t_{0}, \omega\right) \leq \liminf _{n \rightarrow \infty} S_{n}\left(t_{0}, \omega\right) .
$$

Assume in the mean time that (5.2) is satisfied. Then $\alpha_{Z}\left(t_{0}, \omega\right) \leq 1$, which implies that (5.3) holds when $\liminf _{n \rightarrow \infty} S_{n}\left(t_{0}, \omega\right)=1$. We can thus restrict ourselves to the case $\liminf _{n \rightarrow \infty} S_{n}\left(t_{0}, \omega\right)<1$. Suppose, ad absur$d u m$, that $\liminf _{n \rightarrow \infty} S_{n}\left(t_{0}, \omega\right)<\alpha_{Z}\left(t_{0}, \omega\right)$. The function $t \mapsto Z(t, \omega)$ then belongs to a space $C^{\sigma}\left(t_{0}\right)$ for some real $\sigma$ satisfying $\liminf _{n \rightarrow \infty} S_{n}\left(t_{0}, \omega\right)<$ $\sigma$. We can take $\sigma<1$ because of the inclusion (4.14) and the fact that $\liminf _{n \rightarrow \infty} S_{n}\left(t_{0}, \omega\right) \leq b<1$, where $b$ is defined in (2.7). Thus, the definition of $C^{\sigma}\left(t_{0}\right)$ implies that that there is a constant $C_{1}(\omega)>0$ such that the inequality

$$
\begin{aligned}
\left|Z\left(t_{0}+h, \omega\right)-Z\left(t_{0}, \omega\right)\right| & =\left|V\left(t_{0}+h, t_{0}+h, \omega\right)-V\left(t_{0}, t_{0}, \omega\right)\right| \\
& \leq C_{1}(\omega)|h|^{\sigma}
\end{aligned}
$$

holds for all $|h| \leq 1$. By using this last inequality and applying Lemma 5.1 with the compact set $B=\left\{x \in \mathbb{R}^{d},\left|x-t_{0}\right| \leq 1\right\}$, we get

$$
\begin{aligned}
& \left|V\left(t_{0}+h, t_{0}, \omega\right)-V\left(t_{0}, t_{0}, \omega\right)\right| \\
& \quad \leq\left|V\left(t_{0}+h, t_{0}+h, \omega\right)-V\left(t_{0}, t_{0}, \omega\right)\right|+\left|V\left(t_{0}+h, t_{0}+h, \omega\right)-V\left(t_{0}+h, t_{0}, \omega\right)\right| \\
& \quad \leq C_{1}(\omega)|h|^{\sigma}+C(\omega)|h| \leq C_{3}(\omega)|h|^{\sigma},
\end{aligned}
$$

that is, the function $t \mapsto V\left(t, t_{0}, \omega\right)$ belongs to the space $C^{\sigma}\left(t_{0}\right)$. This contradicts the assumption of the proposition, that the pointwise Hölder exponent of this function at $t_{0}$, equals $\liminf _{n \rightarrow \infty} S_{n}\left(t_{0}, \omega\right)$.

Let us now show that (5.2) holds. Assume it does not, that is, for some constant $C_{4}(\omega)>0$ and all $|h|$ small enough, we have $\mid Z\left(t_{0}+h, \omega\right)-$ $Z\left(t_{0}, \omega\right)\left|\leq C_{4}(\omega)\right| h \mid$. Using this inequality and Lemma 5.1 we obtain that for some constant $C_{5}(\omega)>0$ and all $|h|$ small enough, we have $\mid V\left(t_{0}+\right.$ $\left.h, t_{0}, \omega\right)-V\left(t_{0}, t_{0}\right)\left|\leq C_{5}(\omega)\right| h \mid$. This contradicts the assumption that

$$
\limsup _{t \rightarrow t_{0}} \frac{\left|V\left(t, t_{0}, \omega\right)-V\left(t_{0}, t_{0}, \omega\right)\right|}{\left|t-t_{0}\right|}=\infty .
$$

We prove next that

$$
\alpha_{Z}\left(t_{0}, \omega\right) \geq \liminf _{n \rightarrow \infty} S_{n}\left(t_{0}, \omega\right) .
$$

This inequality is clearly satisfied when $\liminf _{n \rightarrow \infty} S_{n}\left(t_{0}, \omega\right)=0$. 
To show that it is also true when $\lim _{n \rightarrow \infty} \inf _{n \rightarrow \infty}\left(t_{0}, \omega\right)>0$, it is sufficient to prove that the function $t \mapsto Z(t, \omega)$ belongs to $C^{s}\left(t_{0}\right)$ for any real $s$ satisfying $0 \leq s<\liminf _{n \rightarrow \infty} S_{n}\left(t_{0}, \omega\right) \leq 1$. Since the function $t \mapsto V\left(t, t_{0}, \omega\right)$ belongs to the space $C^{s}\left(t_{0}\right)$, there is a constant $C_{6}(\omega)>0$ such that the inequality

$$
\left|V\left(t_{0}+h, t_{0}, \omega\right)-V\left(t_{0}, t_{0}, \omega\right)\right| \leq C_{6}(\omega)|h|^{s},
$$

holds for all $|h| \leq 1$. Lemma 5.1 implies

$$
\begin{aligned}
& \left|Z\left(t_{0}+h, \omega\right)-V\left(t_{0}, t_{0}, \omega\right)\right| \\
& \quad=\left|V\left(t_{0}+h, t_{0}+h, \omega\right)-V\left(t_{0}, t_{0}, \omega\right)\right| \\
& \leq\left|V\left(t_{0}+h, t_{0}+h, \omega\right)-V\left(t_{0}+h, t_{0}, \omega\right)\right|+\left|V\left(t_{0}+h, t_{0}, \omega\right)-V\left(t_{0}, t_{0}, \omega\right)\right| \\
& \quad \leq \sup _{x \in B}\left|V\left(x, t_{0}+h, \omega\right)-V\left(x, t_{0}, \omega\right)\right|+C_{4}(\omega)|h|^{s}
\end{aligned}
$$

where $B=\left\{x \in \mathbb{R}^{d},\left|x-t_{0}\right| \leq 1\right\}$. Hence

$$
\left.\mid Z\left(t_{0}+h, \omega\right)-V\left(t_{0}, t_{0}, \omega\right)\right)\left.\left|\leq C_{2}(\omega)\right| h\left|+C_{4}(\omega)\right| h\right|^{s} \leq C_{5}(\omega)|h|^{s},
$$

which proves that the function $t \mapsto Z(t, \omega)$ belongs to the space $C^{s}\left(t_{0}\right)$.

Proof of Theorem 1.1. The result follows from Theorem 1.1 and Proposition 5.1.

\section{Auxiliary results}

The following proposition is the stochastic version of Proposition 1.1 and its proof is structured as the proof of the direct part of Theorem 1 of [15].

Proposition 6.1 Let $\{X(t)\}_{t \in \mathbb{R}^{d}}$ be a stochastic process satisfying the following property: there is $\Omega_{5}^{*}$ an event of probability 1, such that for any $\omega \in \Omega_{5}^{*}$ the path $x \mapsto X(x, \omega)$ is continuous and nowhere differentiable. Set, for any $n \in \mathbb{N}$, any $t \in \mathbb{R}^{d}$ and any $\omega$,

$$
\alpha_{n, X}(t, \omega)=
$$

$=\left\{\begin{array}{l}\min \left(1, \max \left(0, \inf _{2^{-n-2} \leq|h|<2^{-n-1}}\left\{\frac{\log \left(|X(t+h, \omega)-X(t, \omega)|+2^{-n^{2}}\right)}{\log |h|}\right\}\right)\right) \text { if } \omega \in \Omega_{5}^{*}, \\ 0 \text { else. }\end{array}\right.$ 
Then,

(i) For each $n \in \mathbb{N}$ and each $t \in \mathbb{R}^{d}, \alpha_{n, X}(t)$ is a random variable with values in $[0,1]$.

(ii) For every $\omega \in \Omega_{5}^{*}$ and $n \in \mathbb{N}$ the function $t \mapsto \alpha_{n, X}(t, \omega)$ is continuous over $\mathbb{R}^{d}$.

(iii) For all $\omega \in \Omega_{5}^{*}$ and $t \in \mathbb{R}^{d}$,

$$
\alpha_{X}(t, \omega)=\liminf _{n \rightarrow \infty} \alpha_{n, X}(t, \omega),
$$

where $\left\{\alpha_{X}(t)\right\}_{t \in \mathbb{R}^{d}}$ denotes the pointwise Hölder exponent of $\{X(t)\}_{t \in \mathbb{R}^{d}}$.

Proof. We will suppose throughout that $\omega \in \Omega_{5}^{*}$ and we let $\Omega$ will denote the probability space.

Part (i). As the function $x \mapsto X(x, \omega)$ is continuous over $\mathbb{R}^{d}$, for every $t \in \mathbb{R}^{d}$ and every $n \in \mathbb{N}$, one has

$$
\begin{aligned}
& \alpha_{n, X}(t, \omega)= \\
& =\min \left(1, \max \left(0, \inf _{h \in G_{n}}\left\{\frac{\log \left(|X(t+h, \omega)-X(t, \omega)|+2^{-n^{2}}\right)}{\log |h|}\right\}\right)\right),
\end{aligned}
$$

where

$$
G_{n}=\left\{h \in \mathbb{Q}^{d} ; 2^{-n-2} \leq|h|<2^{-n-1}\right\} .
$$

Next, observe that for every $n \in \mathbb{N}$ and $t \in \mathbb{R}^{d}$,

$$
W_{n}(t)=\inf _{h \in G_{n}}\left\{\frac{\left|\log \left(|X(t+h)-X(t)|+2^{-n^{2}}\right)\right|}{|\log | h||}\right\}
$$

is a random variable over $\Omega_{5}^{*}$ since it is the infimum of a sequence of random variables over $\Omega_{5}^{*}$. Relations (6.3) and (6.1) imply therefore that $\alpha_{n, X}(t)$ is a random variable over $\Omega$ with values in $[0,1]$.

Part (ii). It is sufficient to show that the function $t \mapsto W_{n}(t, \omega)$ is continuous over $\mathbb{R}^{d}$, i.e. for any $t_{0} \in \mathbb{R}^{d}$ and $\epsilon>0$, there is $\eta>0$ such that for all $k \in \mathbb{R}^{d}$ satisfying $|k| \leq \eta$, one has

$$
\left|W_{n}\left(t_{0}+k, \omega\right)-W_{n}\left(t_{0}, \omega\right)\right| \leq \epsilon .
$$

Let $g_{n, t_{0}}$ be the function defined over the compact domain

$$
\Delta=\left\{k \in \mathbb{R}^{d} ;|k| \leq 1\right\} \times\left\{h \in \mathbb{R}^{d} ; 2^{-n-2} \leq|h| \leq 2^{-n-1}\right\}
$$

as the function

$$
g_{n, t_{0}}(k, h, \omega)=\left|\log \left(\left|X\left(t_{0}+k+h, \omega\right)-X\left(t_{0}+k, \omega\right)\right|+2^{-n^{2}}\right)\right| .
$$


Observe that one has for every $|k| \leq 1$,

$$
W_{n}\left(t_{0}+k, \omega\right)=\inf _{h \in G_{n}}\left\{\frac{g_{n, t_{0}}(k, h, \omega)}{|\log | h||}\right\}
$$

As the function $g_{n, t_{0}}$ is uniformly continuous over $\Delta$, there is an $\alpha \in(0,1 / 2)$ such that for any $\left(k_{1}, h_{1}\right) \in \Delta$ and $\left(k_{2}, h_{2}\right) \in \Delta$ satisfying $\left|k_{1}-k_{2}\right| \leq \alpha$ and $\left|h_{1}-h_{2}\right| \leq \alpha$, one has

$$
\left|g_{n, t_{0}}\left(k_{1}, h_{1}, \omega\right)-g_{n, t_{0}}\left(k_{2}, h_{2}, \omega\right)\right| \leq \frac{\epsilon}{(n+2) \log 2} .
$$

Consequently, for all $|k| \leq \alpha$ and $2^{-n-2} \leq|h| \leq 2^{-n-1}$,

$$
\frac{\left|g_{n, t_{0}}(k, h, \omega)-g_{n, t_{0}}(0, h, \omega)\right|}{|\log | h||} \leq \epsilon .
$$

Using the last inequality and the fact that $g_{n, t_{0}}$ is a nonnegative function, one obtains that

$$
\frac{g_{n, t_{0}}(k, h, \omega)}{|\log | h||} \leq \epsilon+\frac{g_{n, t_{0}}(0, h, \omega)}{|\log | h||}
$$

and

$$
\frac{g_{n, t_{0}}(0, h, \omega)}{|\log | h||} \leq \epsilon+\frac{g_{n, t_{0}}(k, h, \omega)}{|\log | h||}
$$

which entails that

$$
\inf _{h \in G_{n}} \frac{g_{n, t_{0}}(k, h, \omega)}{|\log | h||} \leq \epsilon+\inf _{h \in G_{n}} \frac{g_{n, t_{0}}(0, h, \omega)}{|\log | h||}
$$

and

$$
\inf _{h \in G_{n}} \frac{g_{n, t_{0}}(0, h, \omega)}{|\log | h||} \leq \epsilon+\inf _{h \in G_{n}} \frac{g_{n, t_{0}}(k, h, \omega)}{|\log | h||} .
$$

Finally, the last two inequalities and (6.8) imply that for every $|k| \leq \alpha$,

$$
\left|W_{n}\left(t_{0}+k, \omega\right)-W_{n}\left(t_{0}, \omega\right)\right| \leq \epsilon .
$$

Thus, taking $\eta=\alpha$ one gets (6.6).

Part (iii). First we show that if the function $x \mapsto X(x, \omega)$ does not belong to a space $C^{\gamma}(t)$ where $\gamma \in(0,1)$, then

$$
\liminf _{n \rightarrow \infty} \alpha_{n, X}(t, \omega) \leq \gamma .
$$

It follows from (4.13) that there is a constant $c_{1}>0$, a sequence $\left(k_{p}\right)_{p \in \mathbb{N}}$ of vectors of $\mathbb{R}^{d}$ and an increasing sequence $\left(n_{p}\right)_{p \in \mathbb{N}}$ of nonnegative integers such that for every $p \in \mathbb{N}$,

(6.10) $2^{-n_{p}-2} \leq\left|k_{p}\right|<2^{-n_{p}-1}$ and $c_{1}\left|k_{p}\right|^{\gamma} \leq\left|X\left(t+k_{p}, \omega\right)-X(t, \omega)\right| \leq 1 / 4$. 
Therefore, one has for every $p \in \mathbb{N}$,

$$
\begin{aligned}
\inf _{2^{-n_{p}-2} \leq|h| \leq 2^{-n_{p}-1}}\left\{\frac{\log \left(|X(t+h, \omega)-X(t, \omega)|+2^{-n_{p}^{2}}\right)}{\log |h|}\right\} \\
\leq \frac{\log \left(\left|X\left(t+k_{p}, \omega\right)-X(t, \omega)\right|+2^{-n_{p}^{2}}\right)}{\log \left|k_{p}\right|} \\
\leq \frac{-\log \left(c_{1}\left|k_{p}\right|^{\gamma}+2^{-n_{p}^{2}}\right)}{\left(n_{p}+1\right) \log 2} \\
\leq \frac{-\log \left(c_{1} 2^{-\left(n_{p}+2\right) \gamma}+2^{-n_{p}^{2}}\right)}{\left(n_{p}+1\right) \log 2}
\end{aligned}
$$

Hence,

$$
\liminf _{p \rightarrow \infty} \inf _{2^{-n_{p}-2} \leq|h| \leq 2^{-n_{p}-1}}\left\{\frac{\log \left(|X(t+h, \omega)-X(t, \omega)|+2^{-n_{p}^{2}}\right)}{\log |h|}\right\} \leq \gamma
$$

and (6.9) results from this last inequality and (6.1).

We now show that if the function $x \mapsto X(x, \omega)$ belongs to a space $C^{\gamma^{\prime}}\left(t_{0}\right)$ where $\gamma^{\prime} \in(0,1)$, then

$$
\liminf _{n \rightarrow \infty} \alpha_{n, X}(t, \omega) \geq \gamma^{\prime}
$$

It follows from (4.13) that there is a constant $c_{2}>0$ such that for every integer $n$ big enough and every $h \in \mathbb{R}^{d}$ satisfying $2^{-n-2} \leq|h|<2^{-n-1}$, one has

$$
|X(t+h, \omega)-X(t, \omega)| \leq c_{2}|h|^{\gamma^{\prime}}<c_{2} 2^{-(n+1) \gamma^{\prime}} \leq 1 / 4 .
$$

This implies that

$$
\frac{\log \left(|X(t+h, \omega)-X(t, \omega)|+2^{-n^{2}}\right)}{\log |h|} \geq \frac{-\log \left(c_{2} 2^{-(n+1) \gamma^{\prime}}+2^{-n^{2}}\right)}{(n+2) \log 2}
$$

and consequently that

$$
\begin{gathered}
\inf _{2^{-n-2} \leq|h| \leq 2^{-n-1}}\left\{\frac{\log \left(|X(t+h, \omega)-X(t, \omega)|+2^{-n^{2}}\right)}{\log |h|}\right\} \\
\geq \frac{-\log \left(c_{2} 2^{-(n+1) \gamma^{\prime}}+2^{-n^{2}}\right)}{(n+2) \log 2} .
\end{gathered}
$$

Then (6.11) follows from this last inequality and (6.1).

Finally, using (6.9), (6.11) and the fact that the function $x \mapsto X(x, \omega)$ is nowhere differentiable (which implies that for every $t \in \mathbb{R}^{d}, \alpha_{X}(t, \omega) \leq 1$ ) one obtains (6.2). 
Proposition 6.2 Let $\{X(t)\}_{t \in \mathbb{R}^{d}}$ be a continuous and nowhere differentiable Gaussian process and $\left\{\alpha_{X}(t)\right\}_{t \in \mathbb{R}^{d}}$ be its pointwise Hölder exponent. Then for any $t_{0}$, the random variable $\alpha_{X}\left(t_{0}\right)$ is deterministic.

Proof of Proposition 6.2. Notice first that as the paths of the process $\{X(t)\}_{t \in \mathbb{R}^{d}}$ are nowhere differentiable with probability 1 , one has almost surely for every $t_{0} \in \mathbb{R}^{d}$,

$$
\alpha_{X}\left(t_{0}\right) \leq 1
$$

Next, fix $t_{0} \in \mathbb{R}^{d}$ and, for every $s \in[0,1]$, denote by

$$
P\left(\alpha_{X}\left(t_{0}\right) \geq s\right)
$$

the probability that the pointwise Hölder exponent at $t_{0}$ of the process $\{X(t)\}_{t \in \mathbb{R}^{d}}$ is greater than $s$. Clearly, one has

$$
P\left(\alpha_{X}\left(t_{0}\right) \geq s\right)=P\left(\bigcap_{n \in \mathbb{N}}\left\{\sup _{t \in B}\left(\frac{\left|X(t)-X\left(t_{0}\right)\right|}{\left|t-t_{0}\right|^{\gamma_{n}}}\right)<\infty\right\}\right),
$$

where $B=\left\{t \in \mathbb{R}^{d},\left|t-t_{0}\right| \leq 1\right\}$ and, for every $n \in \mathbb{N}, \gamma_{n}<\gamma_{n+1}<s$ and $\lim _{n \rightarrow \infty} \gamma_{n}=s$. Moreover,

$$
\begin{aligned}
P\left(\bigcap_{n \in \mathbb{N}}\left\{\sup _{t \in B} \frac{\left|X(t)-X\left(t_{0}\right)\right|}{\left|t-t_{0}\right|^{\gamma_{n}}}<\infty\right\}\right) \\
\quad=\inf _{n \in \mathbb{N}} P\left(\sup _{t \in B} \frac{\left|X(t)-X\left(t_{0}\right)\right|}{\left|t-t_{0}\right|^{\gamma_{n}}}<\infty\right),
\end{aligned}
$$

since

$$
\left(\left\{\sup _{t \in B} \frac{\left|X(t)-X\left(t_{0}\right)\right|}{\left|t-t_{0}\right|^{\gamma_{n}}}\right\}\right)_{n \in \mathbb{N}}
$$

is a decreasing sequence of events.

Next, observe that the zero-one law for Gaussian processes implies that for every $n \in \mathbb{N}$, the probability

$$
P\left(\sup _{t \in B} \frac{\left|X(t)-X\left(t_{0}\right)\right|}{\left|t-t_{0}\right|^{\gamma_{n}}}<\infty\right)
$$

can only be equal to zero or one. Relations (6.14) and (6.15) entail therefore that $P\left(\alpha_{X}\left(t_{0}\right) \geq s\right)=0$ or 1 . If we let

$$
g\left(t_{0}\right)=\sup \left\{s \in[0,1], P\left(\alpha_{X}\left(t_{0}\right) \geq s\right)=1\right\},
$$


then a.s,

$$
\alpha_{X}\left(t_{0}\right)=g\left(t_{0}\right)
$$

Indeed, on one hand a.s,

$$
\alpha_{X}\left(t_{0}\right) \geq g\left(t_{0}\right)
$$

On the other hand, when $g\left(t_{0}\right)=1$, Relations (6.13) and (6.18) entail that almost surely $\alpha_{X}\left(t_{0}\right)=g\left(t_{0}\right)=1$. When $g\left(t_{0}\right)<1$, one has that

$$
g\left(t_{0}\right)=\inf \left\{s \in[0,1], P\left(\alpha_{X}\left(t_{0}\right)<s\right)=1\right\} .
$$

Thus (6.17) follows from (6.16) and (6.18).

By using Proposition 6.2 and Fubini Theorem, we conclude the following:

Corollary 6.1 Let $\{X(t)\}_{t \in \mathbb{R}^{d}}$ be a continuous and nowhere differentiable Gaussian process and $\left\{\alpha_{X}(t)\right\}_{t \in \mathbb{R}^{d}}$ be its pointwise Hölder exponent. Then, there is a non-random function $H(t), t \in \mathbb{R}^{d}$ such that, almost surely, almost everywhere, $\alpha_{X}(t, \omega)=H(t)$.

\section{Conclusion}

We have formed a Generalized Multifractional Processes with Random Exponent (GMPRE) by introducing randomness in the pointwise Hölder exponent of a Generalized Multifractional Brownian Motion (GMBM) and we have showed that Relation (1.12) holds in the strong sense for a GMPRE. This is desirable for the following reasons:

1) It is natural in many situations to model the pointwise Hölder exponent of a real-life signal as a stochastic process. In the case of fully developped turbulence, for example, the dependence on initial conditions, boundary values and the injection of energy is so instable that the Hölder exponent can hardly be viewed as a deterministic function, see [21].

2) For analyzing the irregularity of a signal $\{X(t)\}_{t \in \mathbb{R}^{d}}$ having a very erratic pointwise Hölder exponent, one often uses some multifractal spectrum of $\{X(t)\}_{t \in \mathbb{R}^{d}}$, for example the singularities spectrum $\left\{\theta_{X}(\gamma)\right\}_{\gamma \in[0,1]}$ which is a measure of the geometric repartition of the pointwise Hölder exponents of $\{X(t)\}_{t \in \mathbb{R}^{d}}[4,11,12,20,22,25]$. Namely, for any $\gamma \in[0,1]$ and for any $\omega$, the quantity $\theta_{X}(\gamma, \omega)$ is defined as the Hausdorff dimension of the level set $\left\{t \in \mathbb{R}^{d}, \alpha_{X}(t, \omega)=\gamma\right\}$. In order to obtain information about the singularities spectrum of GMPRE one needs to show that Relation (1.12) holds in the strong sense: almost surely for all $t$. 
3) Because of the randomness of its pointwise Hölder exponent, the construction of a GMPRE with a prescribed singularities spectrum seems to be less difficult than that of a GMBM. Indeed, many results on the almost sure Hausdorff dimensions of the level sets of stochastic processes have been already obtained (see for example $[2,14,27,33]$ ) and, generally speaking, the estimation of these Hausdorff dimensions seems to be more accessible than that of the level sets of deterministic functions.

4) It is quite easy to construct a GMPRE $\{Z(t)\}_{t \in \mathbb{R}^{d}}$ whose singularities spectrum is constant over $(0,1)$ with values in $(d-1, d)$, that is, which satisfies in the strong sense, almost surely for any $\gamma \in(0,1)$,

$$
\theta_{Z}(\gamma, \omega)=d-H
$$

where $H \in(0,1)$ is a constant. This can be done as follows. Monrad and Pitt [33] have proved that the Hausdorff dimensions of the level sets of the FBM $\left\{B_{H}(t)\right\}_{t \in \mathbb{R}^{d}}$ satisfy in the strong sense, almost surely, for any $\gamma \geq 0$,

$$
\operatorname{dim}_{\mathcal{H}}\left\{t \in \mathbb{R}^{d}, B_{H}(t)=\gamma\right\}=d-H .
$$

But, in view of Theorem 1.1, one can construct a GMPRE $\{Z(t)\}_{t \in \mathbb{R}^{d}}$ whose pointwise Hölder exponent equals

$$
Q(t)=\max \left\{0, \min \left(B_{H}(t), 1\right)\right\}, t \in \mathbb{R}^{d},
$$

in the strong sense: almost surely for all $t$. Therefore, (7.2) and (7.3) imply that the singularities spectrum of $\{Z(t)\}_{t \in \mathbb{R}^{d}}$ satisfies (7.1), almost surely, for any $\gamma \in(0,1)$.

5) An interesting feature of the GMPRE process is that it is typically nonGaussian. If it were Gaussian, then according Proposition 6.2, its pointwise Hölder exponent, at a fixed point $t$, would be deterministic.

\section{References}

[1] Abry, P., Flandrin, P., Taqqu, M. S. and Veitch, D.: Wavelets for the analysis, estimation and synthesis of scaling data. In Self-Similar Network Traffic and Performance Evaluation, 39-88. K. Park and W. Willinger, editors. Wiley (Interscience Division), New York, 2000.

[2] Adler, R. J.: The geometry of random fields. Wiley Series in Probability and Mathematical Statistics. John Wiley \& Sons, Ltd., Chichester, 1981.

[3] Andersson, P.: Characterization of pointwise Hölder regularity. Appl. Comput. Harmon. Anal. 4 (1997), 429-443. 
[4] Aubry, J-M. and Jaffard, S.: Random wavelet series. Comm. Math. Phys. 227 (2002), 483-514.

[5] Ayache, A.: The generalized multifractional field: a nice tool for the study of the generalized multifractional Brownian motion. J. Fourier Anal. Appl. 8 (2002), 581-601.

[6] Ayache, A. and Taqqu, M. S.: Multifractional processes with random exponent. Publ. Mat. 49 (2005), 459-486.

[7] Ayache, A. And LÉvy VÉHel, J.: Generalized multifractional Brownian motion: definition and preliminary results. In Fractals: theory and applications in engineering, 17-32. Springer, London, 1999.

[8] Ayache, A. And LÉvy VÉHel, J.: The generalized multifractional Brownian motion. Stat. Inference Stoch. Process. 3 (2000), 7-18.

[9] Ayache, A. And LÉvy VÉhel, J.: On the identification of the pointwise Hölder exponent of the generalized multifractional Brownian motion. Stochastic Process. Appl. 111 (2004), 119-156.

[10] Baccelli, F. AND Hong, D.: AIMD, fairness and fractal scaling of TCP traffic. In Proc. INFOCOM 2002, New York, July 2002.

[11] Barral, J. and Mandelbrot, B. B.: Multifractal products of cylindrical pulses. Probab. Theory Related Fields 124 (2002), 409-430.

[12] Barral, J. And Seuret, S.: From multifractal measures to multifractal wavelet series. J. Fourier Anal. Appl. 11 (2005), 589-614.

[13] Benassi, A., Jaffard, S. And Roux, D.: Elliptic Gaussian random processes. Rev. Mat. Iberoamericana 13 (1997), 19-90.

[14] Berman, S. M.: Gaussian sample functions: Uniform dimension and Hölder conditions nowhere. Nagoya Math. J. 46 (1972), 63-86

[15] Daoudi, K., LÉvy Véhel, J. And Meyer, Y.: Construction of continuous functions with prescribed local regularity. Constr. Approx. 14 (1998), 349-385.

[16] Doukhan, P., Oppenheim, G. and Taqqu, M. S.: Theory and Applications of Long-range Dependence. Birkhäuser, Boston, 2003.

[17] Falconer, K.: Fractal geometry. Mathematical foundations and applications. John Wiley \& Sons, Ltd., Chichester, 1990.

[18] Falconer, K.: Tangent fields and the local structure of random fields. J. Theoret. Probab. 15 (2002), 731-750.

[19] Falconer, K.: The local structure of random processes. J. London Math. Soc. (2) 67 (2003), 657-672.

[20] Fan, A. H.: Multifractal analysis of infinite products. J. Statist. Phys. 86 (1997), 1313-1336.

[21] Frisch, U.: Turbulence: The legacy of A. N. Kolmogorov. Cambridge University Press, 1995. 
[22] Harte, D.: Multifractals: Theory and Applications, Chapman Hall/CRC, Boca Raton, 2001.

[23] Jaffard, S.: Functions with prescribed Hölder exponent. Appl. Comput. Harmon. Anal. 2 (1995), 400-401.

[24] JAFFARD, S.: Some open problems about multifractal functions. In Fractals in Engineering: from theory to industrial applications, 2-18. J. Lévy Véhel, E. Lutton and C. Tricot, editors. Springer, London-Hong Kong, 1997.

[25] Jaffard, S.: The multifractal nature of Lévy processes. Probab. Theory Related Fields 114 (1999), 207-227.

[26] Jaffard, S., Meyer, Y. And Ryan, R.: Wavelets: Tools for Science and Technology. Society for Industrial and Applied Mathematics (SIAM), Philadelphia, PA, 2001.

[27] Kahane, J. P.: Some random series of functions, second edition. Cambridge Studies in Advanced Mathematics 5. Cambridge University Press, Cambridge, 1985.

[28] Kolmogorov, A. N.: Wienersche Spiralen und einige andere interessante Kurven im Hilbertschen Raum. C. R. (Doklady) Acad. URSS (N.S.) 26 (1940), 115-118.

[29] Lemarié, P. G. And Meyer, Y.: Ondelettes et bases hilbertiennes. Rev. Mat. Iberoamericana 2 (1986), 1-18.

[30] Mandelbrot, B. B.: Fractals and Scaling in Finance: Discontinuity, Concentration, Risk. Springer-Verlag, New York Inc., 1997.

[31] Mandelbrot, B. B. and Van Ness, J. W.: Fractional Brownian motions, fractional noises and applications. SIAM Rev. 10 (1968), 422-437.

[32] Meyer, Y., Sellan, F. And Taqqu, M. S.: Wavelets, generalized white noise and fractional integration: the synthesis of fractional Brownian motion. J. Fourier Anal. Appl. 5 (1999), 465-494.

[33] Monrad, D. And Pitt, L. D.: Local nondeterminism and Hausdorff dimension. In Seminar on Stochastic Processes, 1986, 163-189. Progr. Probab. Statist. 13. Birkhäuser Boston, Boston, MA, 1987.

[34] Peltier, R. F. And LÉvy VéHel, J.: Multifractional Brownian motion: definition and preliminary results. Tech. Rep. RR-2645, INRIA. Le Chesnay, France, 1995.

[35] Riedi, R. H. ANd LÉVy VÉHeL, J.: Multifractal properties of TCP traffic: a numerical study. Techical Report RR-3129. INRIA, Rocquencourt, 1997. See also: LÉvy VÉHel, J. AND RIEdi, R.: Fractional Brownian motion and data traffic modelling. In Fractals in Engineering, 185-202. Springer, 1997.

[36] Samorodnitsky, G. and Taqqu, M. S.: Stable Non-Gaussian Processes: Stochastic Models with Infinite Variance, Chapman and Hall, New York, London, 1994. 
[37] Talagrand, M.: Hausdorff measure of trajectories of multiparameter fractional Brownian motion. Ann. Probab. 23 (1995), 767-775.

Recibido: 6 de septiembre de 2005

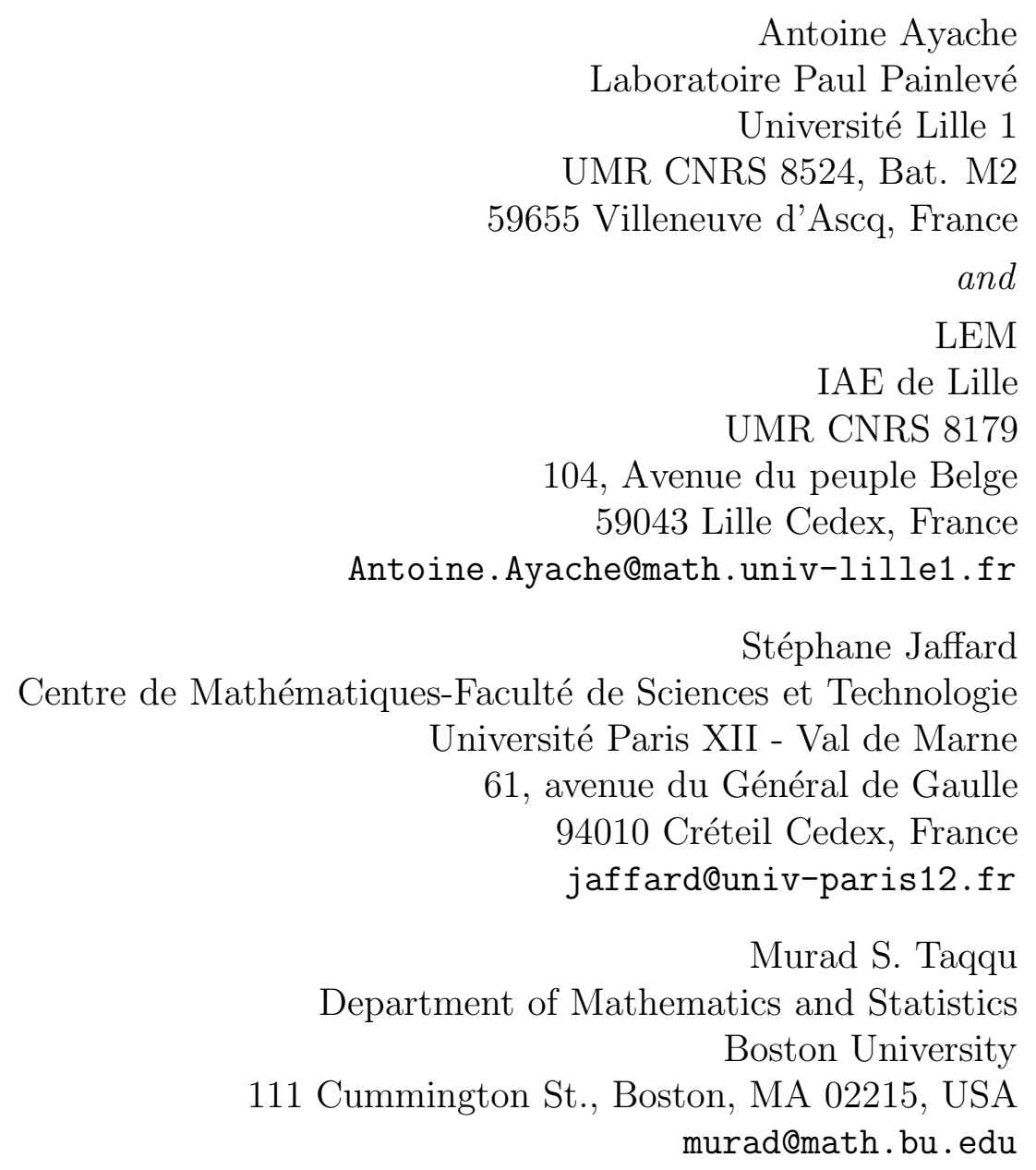

This research was partially supported by the NSF Grants DMS-0102410 and DMS0505747 at Boston University. 